\title{
Oncolytic adenovirus shapes the ovarian tumor microenvironment for potent tumor-infiltrating lymphocyte tumor reactivity
}

.

João Manuel Santos, ${ }^{1,2}$ Camilla Heiniö, ${ }^{1}$ Victor Cervera-Carrascon, ${ }^{1,2}$

Dafne C A Quixabeira, ${ }^{1}$ Mikko Siurala, ${ }^{1,2}$ Riikka Havunen, ${ }^{1,2}$ Ralf Butzow, ${ }^{3}$ Sadia Zafar, ${ }^{1}$ Tanja de Gruijl, ${ }^{4}$ Heini Lassus, ${ }^{5}$ Anna Kanerva, ${ }^{1,5}$

Akseli Hemminki (D) ${ }^{1,2}$

To cite: Santos JM, Heiniö C, Cervera-Carrascon V, et al. Oncolytic adenovirus shapes the ovarian tumor microenvironment for potent tumor-infiltrating lymphocyte tumor reactivity. Journal for ImmunoTherapy of Cancer 2020;8:e000188. doi:10.1136/jitc-2019-000188

- Additional material is published online only. To view please visit the journal online (http://dx.doi.org/10.1136/jitc2019-000188).

Accepted 05 December 2019

Check for updates

(C) Author(s) (or their employer(s)) 2020. Re-use permitted under CC BY-NC. No commercial re-use. See rights and permissions. Published by BMJ.

For numbered affiliations see end of article.

Correspondence to

Dr Akseli Hemminki;

akseli.hemminki@helsinki.fi

\section{ABSTRACT}

Background Ovarian cancers often contain significant numbers of tumor-infiltrating lymphocytes (TILs) that can be readily harnessed for adoptive T-cell therapy (ACT). However, the immunosuppressive ovarian tumor microenvironment and lack of tumor reactivity in TILs can limit the effectiveness of the therapy. We hypothesized that by using an oncolytic adenovirus (Ad5/3-E2F-D24-hTNFa-IRES-hIL2; TILT-123) to deliver tumor necrosis factor alpha (TNFa) and interleukin-2 (IL-2), we could counteract immunosuppression, and enhance antitumor TIL responses in ovarian cancer (OVCA). Methods We established ex vivo tumor cultures freshly derived from patients with advanced OVCA and evaluated the effects of Ad5/3-E2F-D24-hTNFa-IRES-hlL2 or Ad5/3E2F-D24 (the control virus without TNFa and IL-2) on TILs, cytokine response and tumor viability. Tumor reactivity was assessed by determining interferon gamma (IFNg) response of clinically relevant TILs towards autologous T-cell-depleted ex vivo tumor cultures pretreated with or without the aforementioned oncolytic adenoviruses.

Results Treatment of ex vivo tumor cultures with Ad5/3E2F-D24-hTNFa-IRES-hlL2 caused a substantial rise in proinflammatory signals: increased secretion of IFNg, CXCL10, TNFa and IL-2, and concomitant activation of CD4+ and CD8+ TILs. Potent tumor reactivity was seen, as clinically relevant TIL secreted high levels of IFNg in response to autologous T-cell-depleted ovarian ex vivo tumor cultures treated with Ad5/3-E2F-D24-hTNFa-IRES-hIL2. This phenomenon was independent of PD-L1 expression in tumor cells, a factor that determined the variability of IFNg responses seen in different patient samples.

Conclusions Overall, oncolytic adenovirus Ad5/3-E2FD24-hTNFa-IRES-hlL2 was able to rewire the ovarian tumor microenvironment to accommodate heightened antitumor TIL reactivity. Such effects may improve the clinical effectiveness of ACT with TILs in patients with advanced OVCA.

\section{BACKGROUND}

Tumor progression is often mediated by dysfunctional antitumor $\mathrm{T}$ cells in advanced or metastatic solid cancers. ${ }^{1}$ This scenario is evident when disease progression occurs despite the natural existence of tumor-reactive
CD4+ and CD8+ tumor-infiltrating lymphocytes (TIL) at the tumor site. Nevertheless, the existence of naturally occurring TILs has been linked to improved treatment outcome in a number of cancers ${ }^{23}$ posing an opportunity for harnessing tumor-reactive TILs for therapeutic purposes. A potent strategy to achieve this relies on the ex vivo generation and expansion of TILs, for infusion back into the same patient in the setting of adoptive T-cell therapy (ACT). ${ }^{4}$

In heavily pretreated patients with metastatic melanoma, TIL transfer has led to objective response rates up to $70 \%$, and they are regularly seen in about half of patients. ${ }^{5-7}$ Complete responses are seen in about one in four patients, and they tend to be lasting. ${ }^{7}$ However, these responses are yet to be reproduced in other solid tumor indications, including ovarian cancer (OVCA).

The previous clinical data on OVCA TIL therapy are controversial. The reports agree that TILs can be abundant and expansion for therapeutic application is feasible, but clinical efficacy has been variable. One small clinical study reported up to $82 \%$ objective response rate in stage III-IV OVCA. ${ }^{8}$ In other studies, short-term disease stabilization was the best outcome. ${ }^{9} 10$

The presence of an established suppressive network that dampens tumor-specific CD4+ andCD8+ TILs is a feature of the tumor microenvironment of OVCAs, which can decrease the clinical effectiveness of TIL therapy. ${ }^{11}{ }^{12}$ Infiltration by myeloid-derived suppressor cells (MDSC) and regulatory $\mathrm{T}$ cells (Tregs) has been directly linked to poor prognosis in OVCA. $^{213}$ Together, these cell types suppress tumor-specific TIL immunity through a range of mechanisms including, 
but not limited to, the secretion of immunosuppressive cytokines. ${ }^{1314}$ On the other hand, given the mutanome of OVCA, other studies have attributed the lack of efficacy to fewer TIL clones directed against strong tumor-derived antigens, such as neoantigens. ${ }^{10}$

Viral infections are capable of inducing powerful T-cell immunity. ${ }^{15}$ In cancer immunotherapy, this notion has been used to develop therapeutic viruses that replicate and induce immunogenic death in tumor cells, and amplify T-cell-mediated antitumor immunity. ${ }^{16}$ In particular, adenoviruses stand out for their ability to generate new T-cell responses against known tumor antigens ${ }^{17}$ and neoantigens ${ }^{18}$ in cancer-bearing humans, as well as in preclinical models. ${ }^{19}$ Moreover, oncolytic adenoviruses can be modified to harbor transgenes coding for immune stimulatory cytokines, allowing for further customization and amplification of the antitumor immune response.

In the tumor microenvironment of OVCA, the delivery of tumor necrosis factor alpha (TNFa) and interleukin-2 (IL-2) by an oncolytic adenovirus (Ad5/3-E2F-D24hTNFa-IRES-hIL2; TILT-123) could spark a much needed immunological stimulus. This could invigorate TIL activity in the context of ACT, as shown in melanoma and pancreatic cancer. ${ }^{20}$ This is further supported by efficacy data from patient cohorts, where patients with OVCA appeared to benefit from oncolytic adenovirus therapy. ${ }^{21}$ Importantly, among a panel of FDA-approved cytokines, TNFa and IL-2 was the combination of cytokines that best synergized with antitumor $\mathrm{T}$ cells in our preclinical studies. ${ }^{22}{ }^{23}$ This translated into promising safety, efficacy and survival results in animals bearing melanoma and pancreatic tumors, following adoptive transfer of TILs, ${ }^{20}$ T-cell receptor-engineered $\mathrm{T}$ cells ${ }^{22}$ and chimeric antigen receptor $\mathrm{T}$ cells. ${ }^{24}$ Such outcome is attributed to a decrease in intratumororal immunosuppression and, improved trafficking and activation of transferred $\mathrm{T}$ cells. Notably, this approach also seems promising in the context of unleashing $\mathrm{T}$ cells with checkpoint inhibitors. ${ }^{25} 26$

Thus, we studied the possibility of using TNFa and IL-2coding oncolytic adenovirus to restore and enhance the tumor reactivity of TILs in the context of immunosuppressive human OVCA. We established patient-derived ex vivo tumor cultures and studied how the introduction of TNFa and IL-2 by an oncolytic virus, reshapes the overall cytokine response and activation status of TILs in the tumor microenvironment. We then studied the consequences of such immune modulation on the antitumor reactivity of TILs, by transferring clinically relevant TILs into autologous adenovirus-infected human OVCA single-cell suspensions depleted of endogenous T cells.

\section{METHODS \\ Patient material}

OVCA samples were collected from six patients undergoing surgical resection at the Helsinki University Central Hospital (Helsinki, Finland). Almost all patients included in the study were treatment naive, with exception of patient OVCA \#3 who had previously received three rounds of taxane-platinum neoadjuvant chemotherapy prior to surgery (table 1 ).

\section{Oncolytic adenoviruses}

The viruses used in this study were generated using genetic editing strategies previously described by our group. Briefly, Ad5/3-E2F-D24 was generated by cotransfecting PacI/PmeI-linearized pShuttle $\Delta 24$ (plasmid

Table 1 Characteristics of patients with ovarian cancer

\begin{tabular}{|c|c|c|c|c|c|c|}
\hline Patient ID & Age $^{\star}$ & Diagnosis & Pretreatment & Resection & Location & $\begin{array}{l}\text { Tumor percentage } \\
\text { (BRCA mutation } \\
\text { frequency) }\end{array}$ \\
\hline OVCA \#1 & 65 & $\begin{array}{l}\text { Ovarian low-grade serous } \\
\text { carcinoma St IVB }\end{array}$ & - & Metastasis & $\begin{array}{l}\text { Greater } \\
\text { omentum }\end{array}$ & $80 \%$ \\
\hline OVCA \#2P & \multirow[t]{2}{*}{71} & \multirow{2}{*}{$\begin{array}{l}\text { Ovarian high-grade serous } \\
\text { carcinoma St IIIC }\end{array}$} & \multirow[t]{2}{*}{-} & Primary & Ovary & $70 \%$ \\
\hline OVCA \#2M & & & & Metastasis & $\begin{array}{l}\text { Greater } \\
\text { omentum }\end{array}$ & $\mathrm{N} / \mathrm{A}$ \\
\hline OVCA \#3 & 69 & $\begin{array}{l}\text { Ovarian high-grade serous } \\
\text { carcinoma St IVB }\end{array}$ & $\begin{array}{l}\text { Paclitaxel- } \\
\text { carboplatin } \\
\text { (neoadjuvant) }\end{array}$ & Primary & Ovary & $20 \%$ \\
\hline OVCA \#4 & 50 & $\begin{array}{l}\text { Ovarian high-grade serous } \\
\text { carcinoma St IIIC }\end{array}$ & - & Primary & Ovary & $95 \%$ \\
\hline OVCA \#5 & 39 & $\begin{array}{l}\text { Ovarian clear cell carcinoma St } \\
\text { IVB }\end{array}$ & - & Primary & Ovary & $80 \%$ \\
\hline OVCA \#6 & 72 & $\begin{array}{l}\text { Ovarian clear cell carcinoma St } \\
\text { IIIC }\end{array}$ & - & Primary & Ovary & $90 \%$ \\
\hline
\end{tabular}

*at date of resection.

BRCA, breast cancer gene; ID, identification; N/A, not assessed; OVCA, ovarian cancer; St, stage. 
containing E1A with a 24 bp deletion in the CR2 region) with PacI-linearized pTU.5/3 (a plasmid containing an E1A-deleted chimeric ad5 genome) in 911 cells. The resulting plasmid was transfected and propagated in A549 cells. $^{27}$ To generate Ad5/3-E2F-D24-hTNFa-IRES-hIL2, we inserted the cytokine transgenes in the $\mathrm{E} 3$ region of Ad5/3-E2F-D24 using bacterial artificial chromosomerecombineering strategy based on the selection marker galK. ${ }^{20}$ Both viruses were manufactured using a cesium chloride gradient and the concentration was determined by optical density at $260 \mathrm{~nm}$.

\section{Sample processing, establishment of ex vivo and in vitro tumor cultures}

Fresh single-cell tumor digests were prepared from OVCA tumors using a protocol previously validated by our group. ${ }^{28}$ In brief, OVCA tumors were diced into small fragments and placed in a $50 \mathrm{~mL}$ falcon tube containing RPMI 1640 (Sigma-Aldrich, Missouri, USA) supplemented with $1 \%$ L-glutamine, 1\% Pen/strep (Gibco, Thermo Fisher Scientific, Massachusetts, USA), collagenase type I $(170 \mathrm{mg} / \mathrm{L})$, collagenase type IV $(170 \mathrm{mg} / \mathrm{L})$, DNase I $(25 \mathrm{mg} / \mathrm{mL}$ ) and elastase $(25 \mathrm{mg} / \mathrm{mL}$ ) (all enzymes from Worthington Biochemical) for overnight enzymatic digestion with rocking at $+37^{\circ} \mathrm{C}$. After digestion, the cell suspension was filtered through a $100 \mu \mathrm{m}$ filter and treated with Ammonium-Chloride-Potassium lysis buffer (Sigma-Aldrich) for the removal of undigested fragments and red cells. The resulting single-cell suspension was used to establish ex vivo tumor cultures by plating fresh $0.35 \times 10^{6}$ cells in a 96-well plate and treated with multiplicity of infection (MOI; herein defined as virus particle/cell) 100 of Ad5/3-E2F-D24, Ad5/3-E2FD24-TNF-IRES-IL-2 (TILT-123) or medium (vehicle). The remaining tissue was collected in freezing medium containing $90 \%$ fetal bovine serum (FBS) and 10\% dimethyl sulfoxide and stored up to $-140^{\circ} \mathrm{C}$ until further use. Alternatively, human ovarian adenocarcinoma cell line expressing luciferase-firefly SK-OV-3-LUC (kindly provided by Dr Negrin, Stanford Medical School) was established and grown in complete Dulbecco's modified Eagle's medium supplemented with 10\% FBS, 1\% L-glut and $1 \%$ Pen/strep.

\section{Cytotoxicity assay}

The cell viability of OVCA ex vivo tumor cultures was determined in accordance with a protocol previously described by our group. ${ }^{28}$ Briefly, cell viability was verified
A

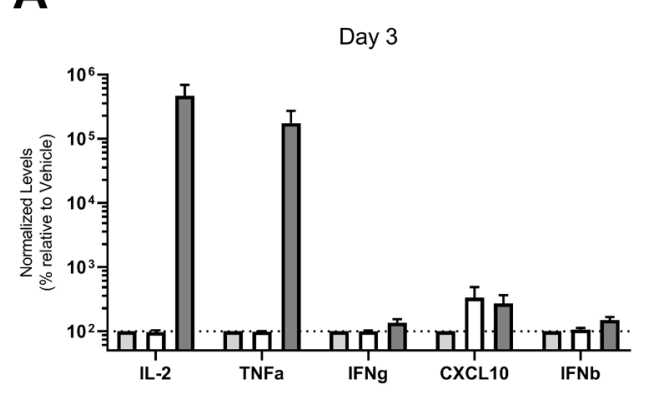

B

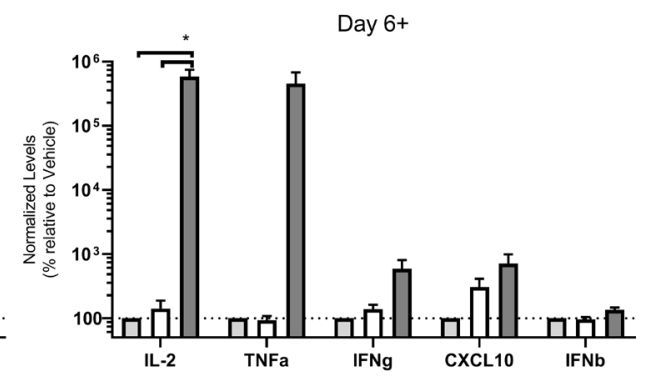

D

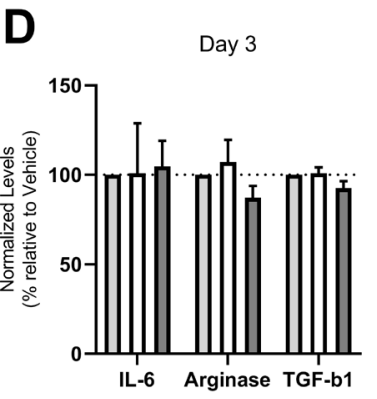

E

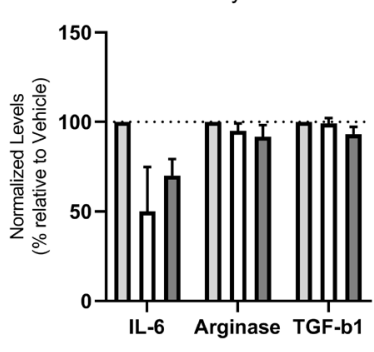

F
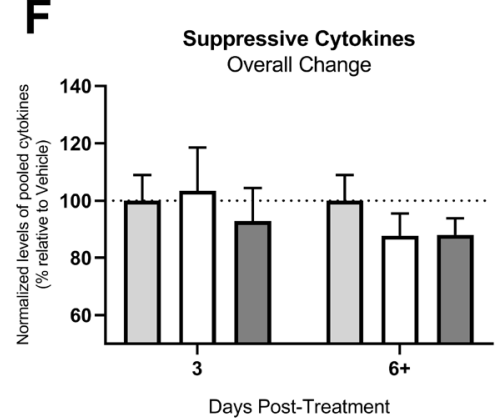

C
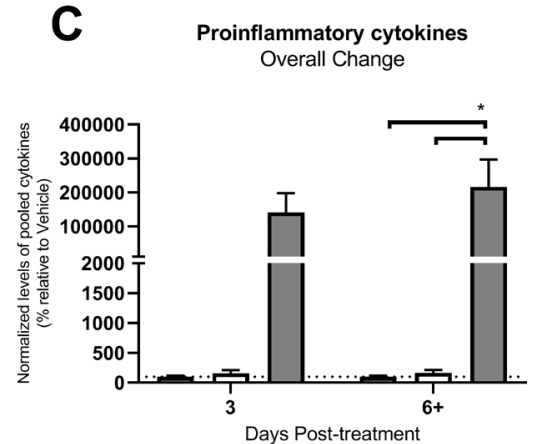

G

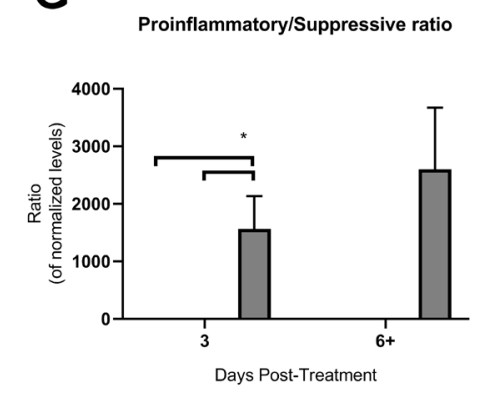

$\square$ Vehicle

ad5/3-E2F-D24

Ad5/3-E2F-D24-hTNFa-IRES-hIL2

Figure 1 Cytokine modulation in ex vivo tumor cultures during oncolytic adenovirus therapy. Supernatants from patient ex vivo tumor cultures were harvested on day 3 and day 6 or 7 for analysis of interleukin-2 (IL-2), tumor necrosis factor alpha (TNFa), interferon gamma (IFNg), C-X-C motif chemokine 10 (CXCL10), interferon beta (IFNb) (proinflammatory cytokines) and interleukin-6 (IL-6), arginase and transforming growth factor beta-1 (TGF-b1) (suppressive cytokines). IL-2 was initially described as T-cell growth factor, while CXCL10 is a potent T-cell recruitment factor. (A) IL-2, TNFa, IFNg, CXCL10, IFNb concentration at day 3 and $(B)$ day 6 or 7 and (C) normalized pooled proinflammatory cytokine changes. (D) IL-6, arginase and TGF-b1 concentration on day 3 and (E) day 6 or 7 and (F) normalized pooled suppressive cytokine changes. (G) Ratio of proinflammatory versus suppressive normalized pooled cytokine subsets. Each patient experiment was performed in quadruplicate and data are shown as mean+SEM. Statistical significance is represented as * $p<0.05$. Dashed line shows the level in vehicle-treated samples (100\%). 
at days 3 and 6 or 7 by incubating 2-3 hours $50 \mu \mathrm{L}$ of CellTiter 96 AQueous One Solution Proliferation Assay reagent (Promega, Wisconsin, USA). Absorbance was read at $492 \mathrm{~nm}$ using a Fluostar OPTIMA analyzer (BMG Labtech, Offenburg, Germany). Each time data point was normalized to the vehicle control group.

\section{Cytokine expression and virus propagation assay}

$0,01 \times 10^{6}$ SK-OV-3-LUC was seeded and incubated overnight in a 96-well plate. The following day different MOIs of Ad5/3-E2F-D24-TNF-IRES-IL-2 were added to culture. After 72 hours, supernatant from cultures was collected in protease inhibitor and stored at $-20^{\circ} \mathrm{C}$ until analysis. Concomitantly, cells were collected in phosphatebuffered saline and their DNA was extracted with QIAmp DNA Mini Kit (Qiagen, the Netherlands) according to the manufacturer's instructions. Virus presence was confirmed by quantitative PCR targeting the d24 deletion of the E1A region using forward primer $\left(5^{\prime}-\mathrm{TCCG}\right.$ GTTTCTATGCCAAACCT- $\left.3^{\prime}\right)$, reverse primer $\left(5^{\prime}\right.$-TCCT CCGGTGATAATGACAAGA- $\left.3^{\prime}\right)$ and probe (5'FAMTGATCGATCCACCCAGTGA-3'MGBNFQ). The results were normalized against the content of human b-actin housekeeping gene DNA, described elsewhere. ${ }^{29}$

\section{Cytokine analysis}

Supernatants from the treated OVCA ex vivo tumor cultures were collected on days 3 and 6 or 7 in the presence or absence of protease inhibitor and stored at $-80^{\circ} \mathrm{C}$. The presence of interferon gamma (IFNg), TNFa, IL-2, interferon beta (IFNb), C-X-C motif chemokine 10 (CXCL10), interleukin 6 (IL-6), arginase, and transforming growth factor beta-1 (TGF-b1) content was determined through a custom LEGENDplex panel and a free active/total TGF-b1 detection kit (Biolegend, California, USA) in agreement with the manufacturer's recommendations. The levels of TNFa and IL-2 in supernatants from SK-OV-3-LUC cells were determined through human IL-2 and TNFa flex sets (BD Biosciences, California, USA). Samples were measured using Accuri C6 flow cytometer (BD Biosciences) and analyzed through LEGENDplex V.8.0 software (VigeneTech, Massachusetts, USA) or FCAP Array software (BD Biosciences). When appropriate, data obtained were normalized to vehicle control group and data points outside of the standard curves were extrapolated according to the software predictions and only considered when at least four values inside the standard curve could be retrieved in one of the analyzed days. The figure 1C,FThe normalized levels of pooled proinflammatory and suppressive cytokines derive from a three-step procedure: (1) each patient's individual cytokine average concentration value of the different therapeutic groups was normalized against the vehicle control, (2) each patient's individual normalized cytokine values were summed according to their category (proinflammatory or suppressive) and the average of each therapeutic group was calculated, and (3) the sum of each patient's grouped cytokines normalized values we renormalized against the vehicle control group. The values shown in the graph are the averages of each therapeutic group.

\section{Generation and expansion of TILs}

TILs were propagated from patient samples using a variation of the 'young TILs' protocol described elsewhere. ${ }^{30}$ Tumor samples were excised into $1-5 \mathrm{~mm}^{3}$ fragments which were placed into six-well G-rex culturing plates (Wilson Wolf, Minnesota, USA) filled with $30 \mathrm{~mL}$ of TIL medium (TM) containing RPMI 1640 supplemented with $20 \% \mathrm{FBS}, 1 \%$ L-glutamine, $1 \%$ Pen/strep, $15 \mathrm{mM}$ HEPES, $1 \mathrm{mM}$ Na-pyruvate, $50 \mu \mathrm{m}$ b-mercaptoethanol (Sigma-Aldrich) and $3000 \mathrm{IU} / \mathrm{mL}$ recombinant human IL-2 (PeproTech, Sweden). Subsequently, the cells were incubated for 5 days in a humidified incubator at $+37^{\circ} \mathrm{C}$. At day 5 , half of the medium was replaced with fresh TM and further incubated until day 7 , in which TILs were collected in freezing medium and stored up to $-140^{\circ} \mathrm{C}$ until further use. Upon collection of five patient tumor samples, $0.8-1.25 \times 10^{6}$ TILs were thawed and rested for 2 days in TM for subsequent rapid expansion. On day 0 , half of the culture medium was replaced with irradiated (40 Gy) allogeneic (four donors) feeder peripheral blood mononuclear cells (PBMC) (1:200 TIL:PBMC ratio), OKT3 antibody (30 ng:mL; eBioscience, Thermo Fisher Scientific) and rapid expansion medium (RM) containing $20 \%$ FBS, $1 \%$ L-glut, $1 \%$ Pen $/$ strep and $3000 \mathrm{IU} / \mathrm{mL}$ recombinant IL-2. On day 5 , half of the medium was replaced by a 1:1 mixture of TM and RM. By day 7, 75\% of the medium was aspirated (while retaining the cells in the bottom) and the number of TILs was adjusted to $5-10 \times 10^{6}$ TILs $/ \mathrm{cm}^{2}$ every $3-4$ days until day 14 .

\section{Autologous TIL-tumor cocultures}

OVCA single-cell suspensions were depleted from CD3+ cells using CD3 MicroBeads (Miltenyi Biotec, Germany) and $0.85 \times 10^{6}$ cells were incubated with MOI 100 of Ad5/3-E2F-D24, Ad5/3-E2F-D24-TNF-IRES-IL-2 (TILT123) or medium in 24-well plates on day 0 . On day 1 , fresh $0.85 \times 10^{6}$ autologous expanded TILs were washed twice with growth medium, added to culture and incubated for 6 additional days in a humidified incubator at $+37^{\circ} \mathrm{C}$. Endpoint supernatant and cells were collected for the detection of IFNg and analysis of T-cell markers, respectively.

\section{IFNg ELISA}

Supernatants from days 2, 5 and 7 of the TIL-tumor cocultures were collected and detection of IFNg was performed using ELISA MAX Deluxe Set Human IFNg (Biolegend) following the manufacturer's instructions. Absorbance was read at $450 \mathrm{~nm}$ using a Fluostar OPTIMA analyzer (BMG Labtech, Offenburg, Germany) and values were interpolated using a line as standard curve with GraphPad Prism V.8software (GraphPad, La Jolla, CA, USA).

\section{Flow cytometry}

Cells from the ex vivo tumor cultures were collected on days 3 and 7 and stained with PE-labelled anti-EpCAM, Alexa 
Fluor 700-labelled anti-CD45, PE/Dazzle 594-labelled anti-CD69, PE-Cy7-labelled anti-programmed death ligand 1 (PD-L1) (Biolegend), PerCP-Cy5.5-labelled anti-CD4, FITC-labelled anti-CD8 and APC-labelled antiCD25 (eBioscience, Thermo Fisher Scientific) according to the manufacturer's instructions. Cells from expanded TILs or autologous TIL-tumor coculture were collected at day 6 and stained with PE-labelled anti-FoxP3, PE/ Dazzle 594-labelled anti-CD3, APC/Cy7-labelled antiCD16 and anti-PD-1, APC-labelled anti-CD56 (Biolegend) and anti-CD25, PerCP-Cy5.5-lablled anti-CD4, FITClabelled anti-CD8 (eBioscience, Thermo Fisher Scientific), PE-Cy7-labelled anti-CD27 (BD Biosciences). A total of 10,000-100,000 events were acquired using FACS Aria II cell sorter (BD Biosciences) and data analysis was performed using FlowJo software V.10 (FlowJo, BD Biosciences). Geometric mean data from activation markers were normalized to the vehicle group.

\section{Histopathology}

After resection, OVCA tissue was fixed, paraffin embedded and cut into 4-5 $\mu \mathrm{m}$ slides either for $\mathrm{H} \& \mathrm{E}$ staining or immunohistochemistry. Anti-PD-L1 (Abcam, UK) was stained using Lab Vision Autostainer (Thermo Fisher Scientific). Anti-CD8 (Novocastra, Leica Biosystems, Illinois, USA) and anti-CD4 (Cell Marque, Merck, California, USA) were stained using a VENTANA Autostainer (Roche, Switzerland). PD-L1 expression was scored based on the frequency of PD-L1+ tumor cells. The score is as follows: $0=<1 \%, 1=1 \%-4 \%, 2=5 \%-9 \%, 3=10 \%-49 \%, 4=>50 \%$ of

A

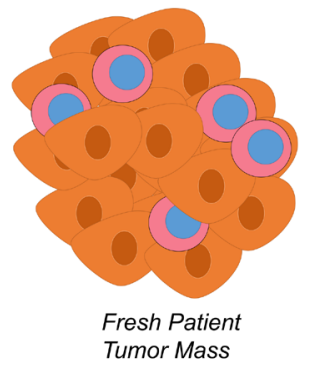

B

OVCA \#2M

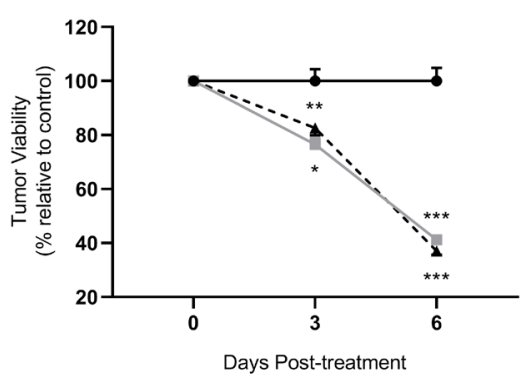

E

OVCA \#4

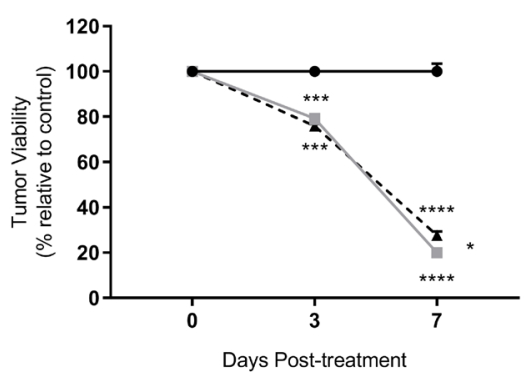

Digestion + Oncolytic Adenovirus Infection

Tumor microenvironment remodeling analysis

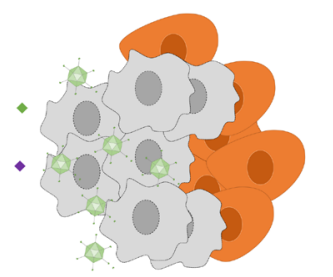

Oncolytic Adenovirus/ OVCA cell suspension culture

\section{Supernatant collection}

Cytokine Multiplex Analysis

D
OVCA \#2P

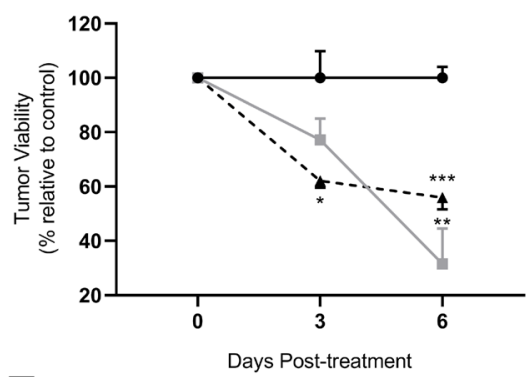

$\mathbf{F}$

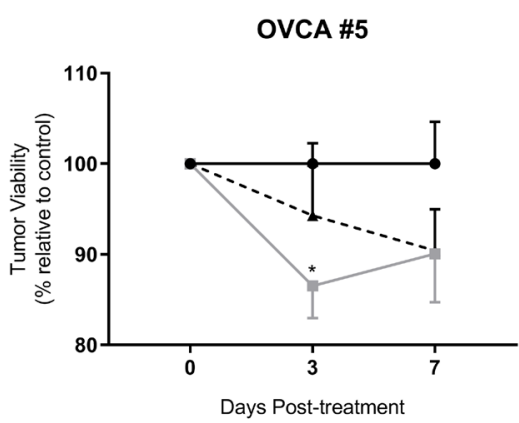

OVCA \#3

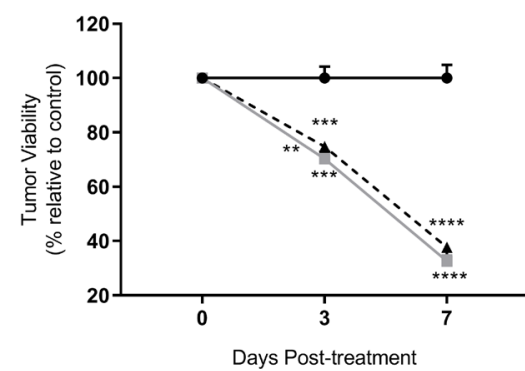

G

OVCA \#6

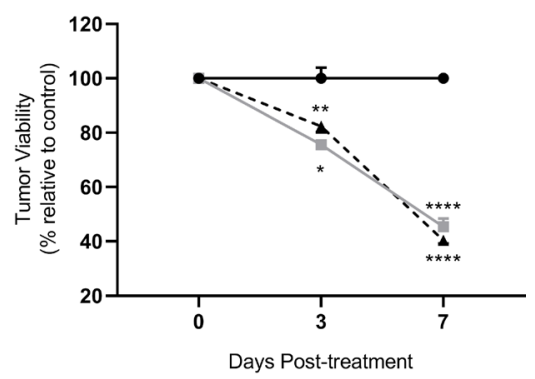

Vehicle Ad5/3-E2F-D24

-^• Ad5/3-E2F-D24-hTNFa-IRES-hIL2

Figure 2 Lytic capability of oncolytic adenovirus in ovarian cancer patient ex vivo tumor cultures. (A) Tumor digests (containing cancer cells, stromal cells and immune cells) were obtained fresh from patients with resected ovarian cancer and cultured for 6-7 days with multiplicity of infection (MOI) 100 of oncolytic virus. Viability was assessed on the indicated days. (B) OVCA \#2M, (C) OVCA \#2P, (D) OVCA \#3, (E) OVCA \#4, (F) OVCA \#5 and (G) OVCA \#6. Each patient experiment was performed in quadruplicate and data are shown as mean+SEM. Statistical significance is represented as ${ }^{*} p<0.05,{ }^{\star \star} p<0.01$, ${ }^{\star \star *} p<0.001$, and ${ }^{* \star \star \star} p<0.0001$. OVCA, ovarian cancer. 
PD-L1+ tumor cells. CD8+ and CD4+ cells were counted using high-power field at 400×. Tumor histologies, breast cancer (BRCA) gene mutated tumor cell frequencies, CD8+ and CD4+ cell count and PD-L1 scores were provided and confirmed by a gynecological pathologist.

\section{Statistical analysis}

Differences between treatment groups were studied by unpaired Student's t-test with Welch's correction while correlations between variables were investigated using Spearman non-parametric correlation, both using GraphPad Prism V.8 software (GraphPad, La Jolla, CA, USA).

\section{RESULTS \\ Oncolytic adenovirus therapy reduces the viability of OVCA patient ex vivo tumor cultures}

We have previously shown that Ad5/3-E2F-D24-hTNFaIRES-hIL2 is able to replicate and lyse human OVCA cell lines. ${ }^{20}$ However, it remained unclear how this phenomenon is affected by virus-derived cytokines and cell subsets present in the tumor microenvironment of different types and stages of OVCA. Therefore, ex vivo tumor cultures from five patients with OVCA bearing tumors with diverse histologies were established up to 7 days (figure 2A). Treatment with Ad5/3-E2F-D24 (control without transgenes) or Ad5/3-E2F-D24-hTNFa-IRES-hIL2 exerted similar antitumor killing profiles in most patient ex vivo tumor cultures.

By day 3, Ad5/3-E2F-D24 or Ad5/3-E2F-D24-hTNFaIRES-hIL2 had significantly decreased the viability of most cultures to values ranging from $95 \%$ to $60 \%$ relative to the vehicle control. In detail, Ad5/3-E2F-D24 was more effective in killing ex vivo tumor cultures from samples OVCA \#3 $(\mathrm{p}<0.01)$ and OVCA \#5 as compared with Ad5/3-E2FD24-hTNFa-IRES-hIL2 (figure 2B). However, the latter was capable of decreasing the viability of OVCA \#2P ex vivo tumor cultures slightly faster than Ad5/3-E2F-D24 therapy. Such difference is inverted by day 6 , when treatment with Ad5/3-E2F-D24 drops the viability of the ex vivo tumor culture to $30 \%$ compared with $60 \%$ in the Ad5/3E2F-D24-hTNFa-IRES-hIL2 therapy group (figure 2B). A similar scenario is observed on day 7 in OVCA \#4 ex vivo tumor cultures where a marginal reduction, although statistically significant $(\mathrm{p}<0.01)$, was more prominent in wells treated with Ad5/3-E2F-D24 relative to Ad5/3E2F-D24-hTNFa-IRES-hIL2 treated wells (figure 2B). Surprisingly, therapy with Ad5/3-E2F-D24 or Ad5/3-E2FD24-hTNFa-IRES-hIL2 was only able to cause reduction of $10 \%$ viability in OVCA \#5 ex vivo tumor culture, which could reflect the limitations of the assay (figure 2B). Of note, the virus is only able to kill malignant cells, while normal cells including TILs would remain unharmed.

\section{Cytokine-coding adenovirus therapy induces extensive immunostimulatory modulation in the tumor microenvironment}

Over 6-7 days of culturing period, treatment of OVCA patient ex vivo cultures with cytokine-coding oncolytic adenoviruses caused changes in the immunological status of the tumor microenvironment, which was reflected in the production of several cytokines. This is possible because OVCA cells can manufacture IL-2 and TNFa. We saw a dose-dependent increase in the levels of these cytokines (online supplementary figure 1A) and virus DNA (online supplementary figure 1B) when we infected an ovarian adenocarcinoma cell line with increasing amounts of Ad5/3-E2F-D24-hTNFa-IRES-hIL2. Furthermore, a significant positive correlation was found between the levels of DNA and TNFa (online supplementary figure 1C; $\mathrm{r}=0.9821$ (0.9438 to 0.9944), $\mathrm{p}<0.0001)$ and IL-2 (online supplementary figure $1 \mathrm{D} ; \mathrm{r}=0.9491 \quad(0.8454$ to 0.9838$)$, $\mathrm{p}<0.0001)$. Hence, during infection with Ad5/3-E2F-D24hTNFa-IRES-hIL2, the levels of these cytokines were very high on day 3 as compared with the vehicle or Ad5/3E2F-D24 treated groups (figure 1A). These differences remain consistent on day $6+$ (figure 1B), with a slight increase in both cytokines relative to day 3. Ad5/3-E2FD24-hTNFa-IRES-hIL2 therapy also induced the production of IFNg and CXCL10 which, in contrast with the vehicle and Ad5/3-E2F-D24 treated groups, had a peak in production at day $6+$ (figure 1B).

Of note, production of CXCL10 by day $6+$ had increased in all therapeutic groups (figure 1B). Interestingly, treatment with oncolytic adenovirus caused only a negligible increase in IFNb production, although levels were steadily higher in groups treated with Ad5/3-E2FD24-hTNFa-IRES-hIL2 compared with the vehicle and Ad5/3-E2F-D24. Thus, the overall presence of proinflammatory signals in patient ex vivo tumor cultures treated with cytokine-coding adenoviruses differed significantly from vehicle and Ad5/3-E2F-D24 on day 3 (1407-fold and 874-fold differences, respectively) and day $6+(2256-$ fold and 1350-fold differences, respectively, both $\mathrm{p}<0.05$ ) (figure 1C). This pool of proinflammatory (with TNFa and IL-2) signals was found to be negatively correlated with tumor histoculture viability $(\mathrm{r}=-0.6427 \quad(-0.8055$ to -0.3897$), \mathrm{p}<0.0001)$. Because TNFa and IL-2 may be from viral origin, we considered excluding these from the proinflammatory cytokine pool. Still, excluding the levels of these cytokines from the proinflammatory pool (thus only with IFNg, CXCL10, IFNb) the ratio was still high on day 6+, in cultures treated with Ad5/3-E2F-D24-hTNFaIRES-hIL2, as compared with the unarmed virus or vehicle control group (2.5-fold and 5.1-fold differences, respectively) (online supplementary figure $1 \mathrm{E}$ ).

In contrast, fewer differences were observed in the levels of factors with suppressive potential, such as IL-6, arginase and TGF-b1. On day 3, treatment with Ad5/3E2F-D24-hTNFa-IRES-hIL2 increased IL-6 relative to the vehicle and Ad5/3-E2F-D24 which stayed at approximately the same level (figure 1D). Conversely, Ad5/3-E2F-D24 and Ad-5/3-E2F-D24-hTNFa-IRES-hIL2 treatment lowered IL-6 relative to the vehicle control (figure 1E). In comparison with vehicle and Ad5/3-E2F-D24, a trend for reduced presence of arginase and TGF-b1 was found in ex vivo tumor cultures treated with cytokine-coding 
oncolytic adenoviruses on days 3 and $6+$. In the latter, a total reduction of suppressive signals was observed on oncolytic adenovirus therapy compared with the vehicle control (figure 1F).

No correlation was found between pooled suppressive cytokines and the reduction of tumor ex vivo tumor culture viability. In the Ad5/3-E2F-D24-hTNFa-IRES-hIL2 group, these changes resulted in a proinflammatory/suppressive ratio of 2713.42, compared with approximately 2.1 and 1.0 for Ad5/3-E2F-D24 and vehicle control, respectively, on day 6+ (figure 1G). These differences were observed to a lower extent when TNFa and IL-2 were excluded from the pooled levels (online supplementary figure 1F).

\section{Activation of CD4+ and CD8+ TILs is improved during virotherapy-mediated immune modulation}

The tumor microenvironment of human OVCA is distinctly characterized by the presence of inactivated TILs which can be readily harnessed if an appropriate stimulus is introduced. Here, treatment of patient ex vivo tumor cultures with TNFa and IL-2 coding oncolytic adenoviruses induced several changes in TIL activation that were not observed in other treatment groups. Comparative to vehicle and Ad5/3-E2F-D24, the use of cytokine-coding oncolytic adenovirus increased the overall presence of CD69 in CD8+ TILs (online supplementary figure 2A), in agreement with statistically significant increases observed in three out of five samples on day 6+ (figure 3A). On the same day, CD8+ TILs expressed higher levels of CD69 in groups treated with oncolytic adenoviruses relative to the vehicle control, which was particularly notable in the Ad5/3-E2F-D24 treatment group on day 6+ (online supplementary figure 2B). When analyzed sample by sample, increase in CD69 expression in CD8+ TILs was only seen for OVCA \#5, in which the difference was statistically significant (Ad5/3-E2F-D24 vs Ad5/3-E2F-D24hTNFa-IRES-hIL2, $\mathrm{p}<0.05$ ). For the majority of samples only a trend for its increased expression was seen on Ad5/3-E2F-D24-hTNFa-IRES-hIL2 therapy (figure 3B). Overall, expression of CD69 in CD8+ TILs was not associated with the secretion of proinflammatory cytokines $(\mathrm{r}=0.007274$ ( -0.5192 to 0.5297$), \mathrm{p}=0.9844$; figure $3 \mathrm{C})$.

With regard to the presence of CD4+CD69+ TILs, on day 6+, a mild reduction was observed during oncolytic adenovirus therapy in comparison with the vehicle control group (online supplementary figure 2C). Although not statistically significant, this reduction was more prominent in the Ad5/3-E2F-D24 treated group (three out of five samples) followed by Ad5/3-E2F-D24hTNFa-IRES-hIL2 treatment group (figure 3D). The latter induced CD69 expression in CD4+ TILs on days 3 and 6 as opposed to the vehicle and Ad5/3-E2F-D24 (online supplementary figure 2D). Similarly, the upregulation of CD69 in CD4+ TILs was statistically higher in three out of five samples following infection with Ad5/3E2F-D24-hTNFa-IRES-hIL2 (figure 3E). Interestingly, this upregulation was positively correlated with the presence of proinflammatory cytokines $(r=0.8547$ ( 0.5985 to
0.9523), $\mathrm{p}<0.0001$; figure $3 \mathrm{~F}$ ), and may be associated with a helper phenotype of these cells.

Concerning the percentage of CD4+CD25+ TILs, an overall increase in this subset was observed on day $6+$, however, the increase was more prominent when no treatment was applied (online supplementary figure 2E). In particular, Ad5/3-E2F-D24-hTNFa-IRES-hIL2 therapy significantly reduced this subset in three out of five samples while Ad5/3-E2F-D24 therapy induced such reduction in two out of five samples (figure $3 \mathrm{G}$ ). Overall, the highest upregulation of CD25 was achieved on day 3 during Ad5/3-E2F-D24 therapy (online supplementary figure 3F), and on day 6 during Ad5/3-E2F-D24-hTNFaIRES-hIL2 (figure 3H). At a sample level, this trend was seen in two out of five patients on day $6+$ (online supplementary figure $2 \mathrm{~F}$ ). These cells were not correlated with suppressive cytokines $(\mathrm{r}=0.1818 \quad(-0.3788$ to 0.6448$)$, $\mathrm{p}=0.5163$; figure $3 \mathrm{I}$ ).

\section{Antitumor reactivity of clinically relevant TILs is improved by cytokine-coding oncolytic adenovirus}

Considering the shift in the immune status of the tumor microenvironment on cytokine-coding oncolytic adenovirus treatment, we set out to evaluate whether such modulation positively favors antitumor reactivity in the context of TIL therapy. Thus, CD3-depleted single-cell suspensions treated with or without oncolytic adenovirus were cocultured with autologous expanded TILs from patients with OVCA (figure 4A). The TIL product contained a CD3+ fraction which comprised CD8+ TILs in its majority (65.5\%), followed by CD4+ TILs (26.4\%) and CD3+CD56+ natural killer (NK) T cells (3.3\%). The CD3fraction included $14.8 \% \mathrm{NK}$ cells, at day 14 (figure $4 \mathrm{~B}$ ). The culturing conditions also allowed the presence of CD4+CD25+FoxP3+ Tregs, with their frequency reaching a maximum of $1.78 \%$ in OVCA \#1 TILs and a minimum of $0.29 \%$ in OVCA \#2M, in the CD4+ fraction (online supplementary figure 3A). The presence of $\mathrm{CD} 27+$ TILs was significantly enriched in the CD8+ fraction as compared with the $\mathrm{CD} 4+$ fraction $(\mathrm{CD} 3+\mathrm{CD} 8+\mathrm{CD} 27+$ vs $\mathrm{CD} 3+\mathrm{CD} 4+\mathrm{CD} 27+; \mathrm{p}<0.005)$. No differences were found regarding CD62L+ TILs (figure 4C).

Following the addition of autologous TILs, IFNg production was highest in two out of six samples in response to Ad5/3-E2F-D24-hTNFa-IRES-hIL2 (figure 4D) on day 2. IFNg peaked for the same samples (OVCA \#2M and OVCA \#4) on day 5 , during a time which new statistically relevant antitumor reactivity emerged in two additional samples (OVCA 3 and OVCA \#5, both $\mathrm{p}<0.05$, figure 4E). A similar situation occurred in OVCA \#2P on day 7 , where, compared with Ad5/3-E2F-D24 treatment, Ad5/3E2F-D24-hTNFa-IRES-hIL2 therapy induced new statistically $(\mathrm{p}<0.05)$ relevant reactivity (figure $4 \mathrm{~F})$. Additionally, during Ad5/3-E2F-D24-hTNFa-IRES-hIL2 therapy, differences in IFNg production became more apparent in sample OVCA \#3, due to the significant reduction of IFNg secretion in the vehicle control $(\mathrm{p}<0.01)$ and Ad5/3E2F-D24 $(\mathrm{p}<0.01)$ groups. This indicated persistent 
A

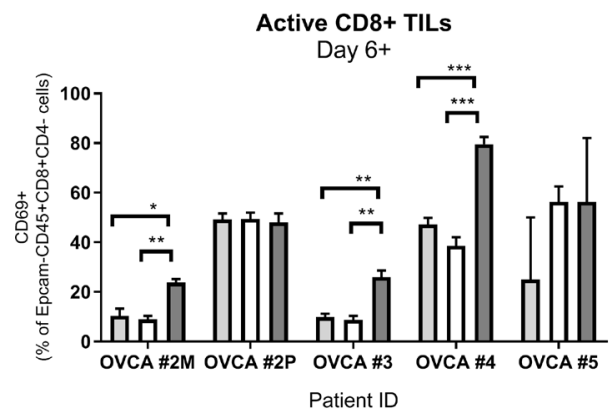

D

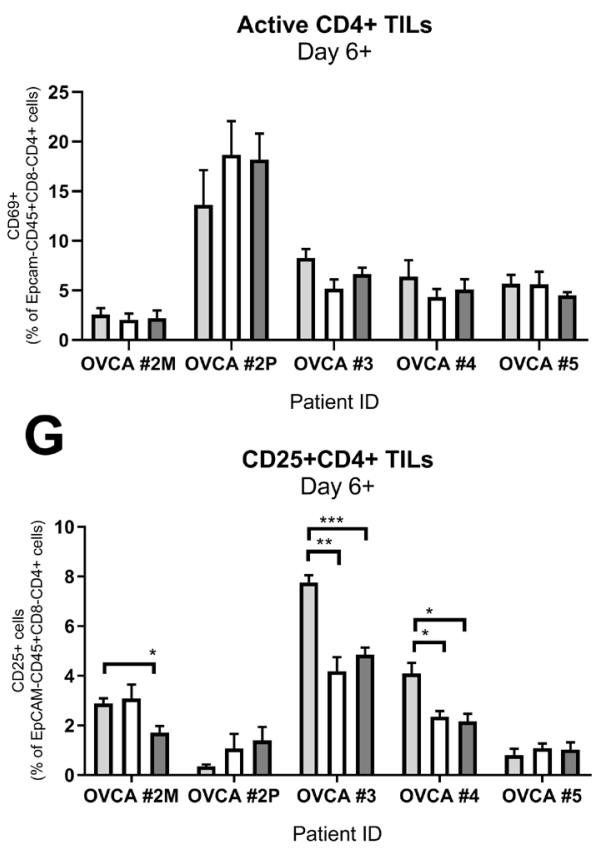

B

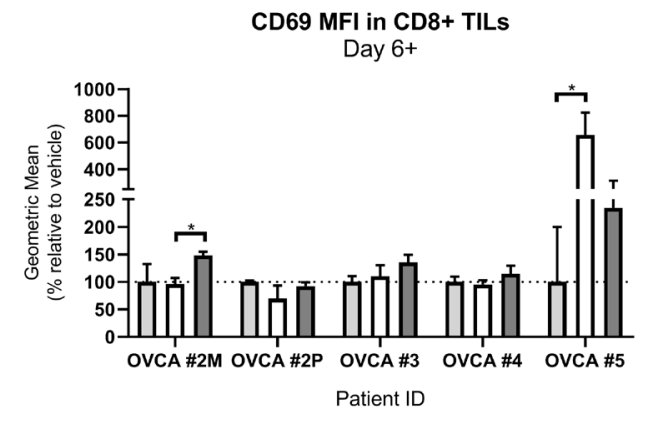

E
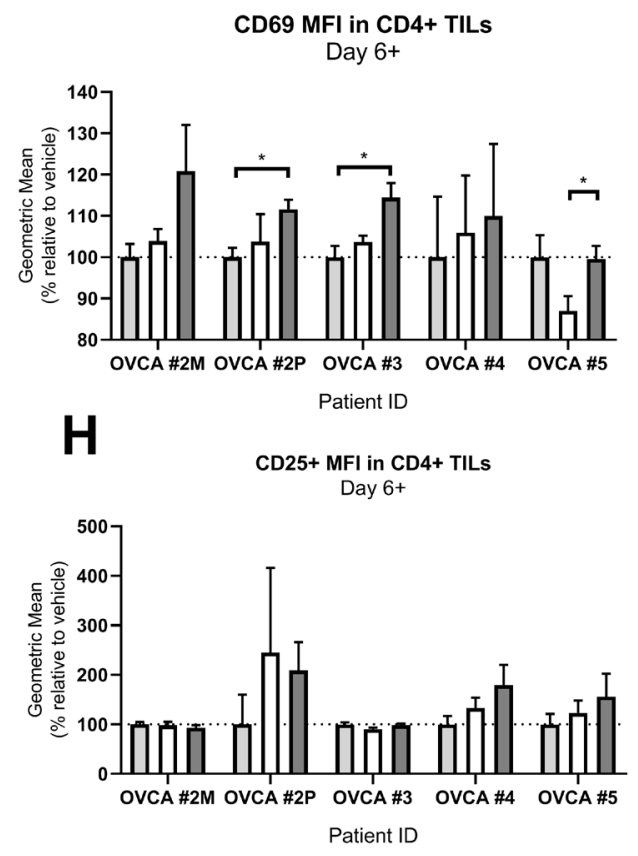

C

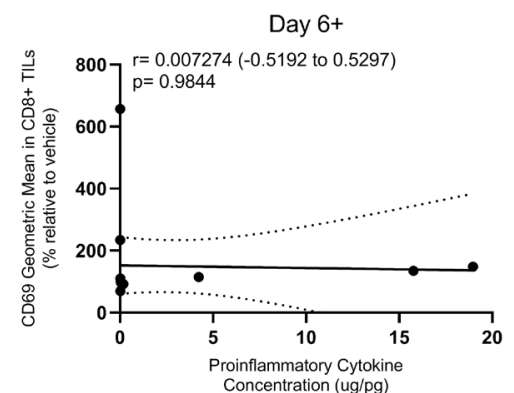

$\mathbf{F}$

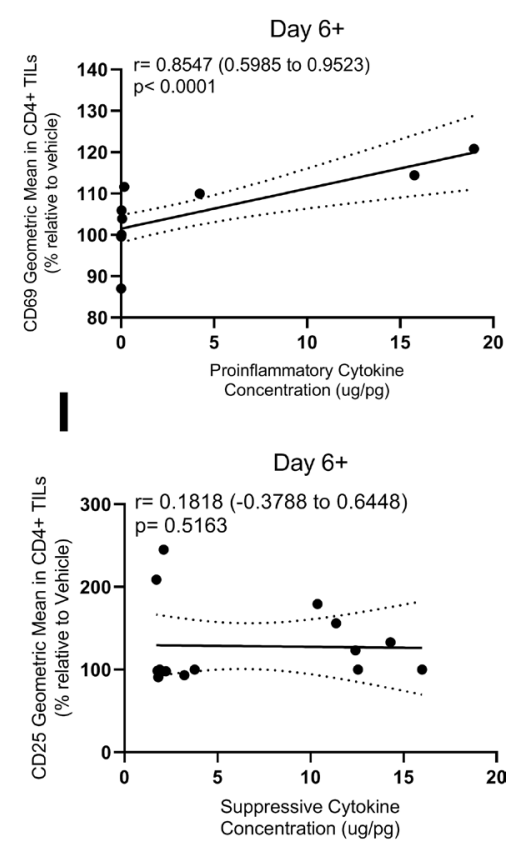

ש Vehicle

Ad5/3-E2F-D24

Ad5/3-E2F-D24-hTNFa-IRES-hIL2

Figure 3 Presence and activation status of CD4+ and CD8+ tumor-infiltrating lymphocytes (TIL) in ex vivo tumor cultures at day 6. Cells from patient ex vivo tumor cultures were harvested on day 6 for analysis of CD69 (an early activation marker) and CD25 (interleukin-2 (IL-2) receptor) in CD8+ and CD4+ TILs. (A) Percentage of CD8+CD69+ TILs, (B) normalized mean fluorescence intensity (MFI; a measure of number of receptors at the surface of gated cells) of CD69 in CD8+ TILs, (C) correlation between CD69 MFI in CD8+ TILs and pooled proinflammatory cytokines from all therapeutic groups, (D) percentage of CD4+CD69+ TILs, (E) normalized MFI of CD69 in CD4+ TILs, (F) correlation between CD69 MFI in CD4+ TILs and pooled proinflammatory cytokines, (G) percentage of CD4+CD25+ TILs, (H) normalized MFI of CD25 in CD4+ TILs, (I) correlation between CD25 MFI in CD4+ TILs and pooled suppressive cytokines. Each patient experiment was performed in quadruplicate and data are shown as mean+SEM. Statistical significance is represented as ${ }^{*} p<0.05,{ }^{* *} p<0.01$ and ${ }^{* * *} p<0.001$. OVCA, ovarian cancer.

stimulatory effects of cytokine-coding oncolytic adenoviruses on TILs (figure 4F). Of note, no statistically relevant differences between treatment groups were found for sample OVCA \#1, over the course of 7 days' coculture. However, during the coculturing period, the antitumor reactivity of autologous TILs was enhanced on Ad5/3-E2FD24-hTNFa-IRES-hIL2 therapy in five out of six samples. Despite the presence of Tregs after expansion of autologous TILs, we did not see a difference in the magnitude of IFNg response in the cocultures $(\mathrm{r}=0.03449(-0.4515$ to 0.5046 ), $\mathrm{p}=0.8919$; online supplementary figure $3 \mathrm{~B}$ ).

\section{Virus-induced enhanced antitumor reactivity associates with CD4+ TILS}

In order to identify the source of the antitumor reactivity seen, we set out to characterize the transferred TILs present in the TIL/tumor cocultures. By day 7 , Ad5/3-E2F-D24-hTNFa-IRES-hIL2 therapy had substantially increased the presence of CD8+ TILs over the vehicle control and Ad5/3-E2F-D24 treatment groups in sample OVCA \#1, OVCA \#3 and OVCA \#4 (figure 5A). In sample OVCA \#2P, this phenomenon extended to the Ad5/3-E2F-D24 treatment group, where the increase was 
A
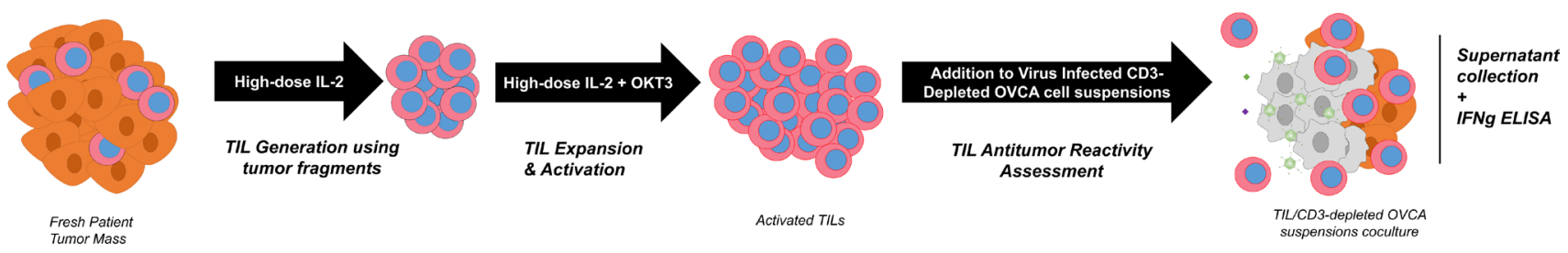

B

\section{TIL Characteristics}

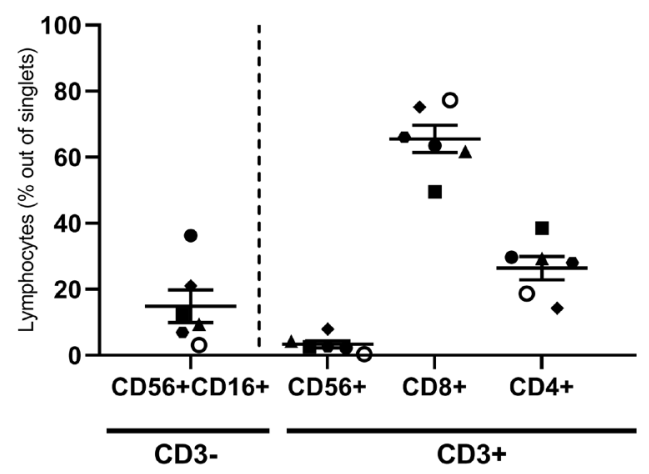

TIL Differentiation Markers

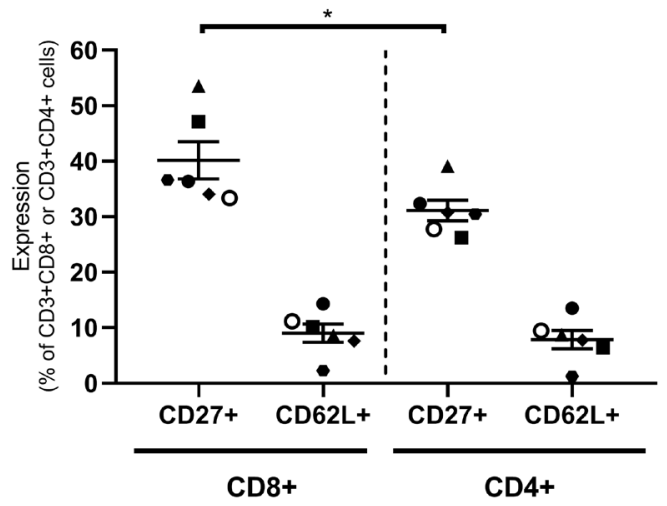

D

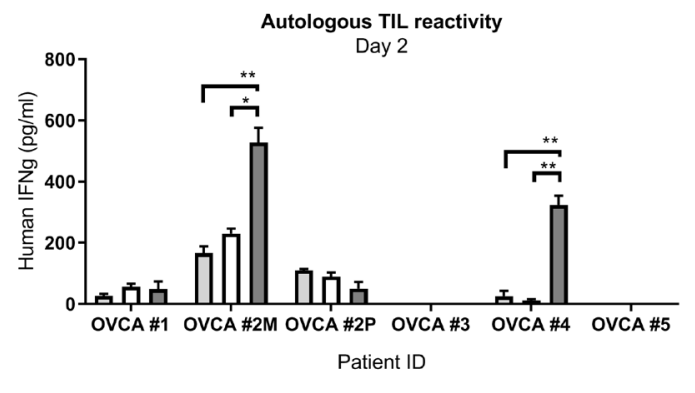

$\mathbf{E}$

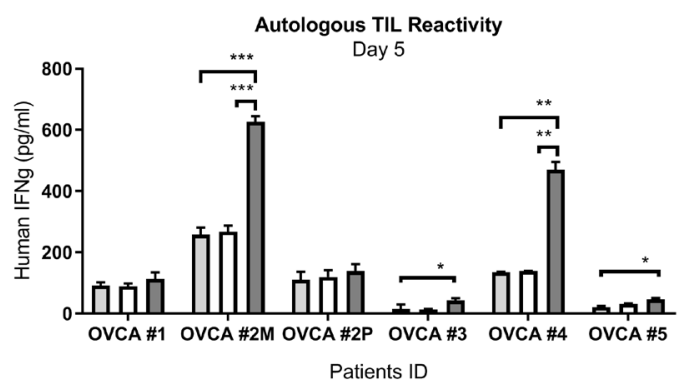

$\mathbf{F}$

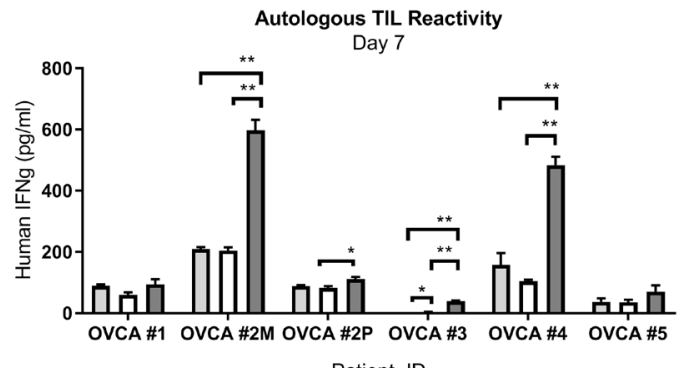

Figure 4 Characterization of expanded tumor-infiltrating lymphocytes (TIL) and reactivity assessment towards autologous tumor digests. (A) Excised tumor fragments from each patient were used for the generation and rapid expansion of TILs during 14 days. These were subsequently cocultured with CD3-depleted autologous tumor digests in the presence or absence of oncolytic adenovirus and supernatants were collected for interferon gamma (IFNg) analysis. (B) Identification of CD3+CD8+ T cells, CD3+CD4+ T cells and CD56+CD16+ natural killer (NK) cells and (C) differentiation status markers, such as CD27 (costimulatory molecule) and CD62L (adhesion molecule for secondary lymphoid structures). (D) Day 2 coculture IFNg levels. (E) Day 5 coculture IFNg levels. (F) Day 7 coculture IFNg levels. Each patient experiment was performed in triplicates and data are shown as mean+SEM. Statistical significance is represented as ${ }^{*} p<0.05,{ }^{* *} p<0.01$ and ${ }^{* * *} p<0.001$. Symbols are as follows: dots-OVCA \#1, squares-OVCA \#2M, triangles-OVCA \#2P, diamonds-OVCA \#3, hexagons-OVCA \#4, hollow dots-OVCA \#5. OVCA, ovarian cancer.

significant compared with the vehicle control group, and as high as in the Ad5/3-E2F-D24-hTNFa-IRES-hIL2 group (figure 5A). In the latter group, the presence of CD27 was statistically reduced $(\mathrm{p}<0.05)$ in OVCA \#1 compared with the vehicle control group (online supplementary figure 3C). Intriguingly, the increase of IFNg content in the wells strongly correlated with the decrease in the presence of CD8+ TILs ( $\mathrm{r}=-0.4223$ ( -0.6249 to -0.1663$)$, $\mathrm{p}=0.0015$; figure $5 \mathrm{~B}$ ).

In a similar manner, the presence of CD4+ TILs in Ad5/3-E2F-D24-hTNFa-IRES-hIL2 treated wells was significantly higher relative to vehicle control and Ad5/3E2F-D24 in samples OVCA \#2M, OVCA \#3 and OVCA \#4. The same trend, although not statistically relevant, 
A

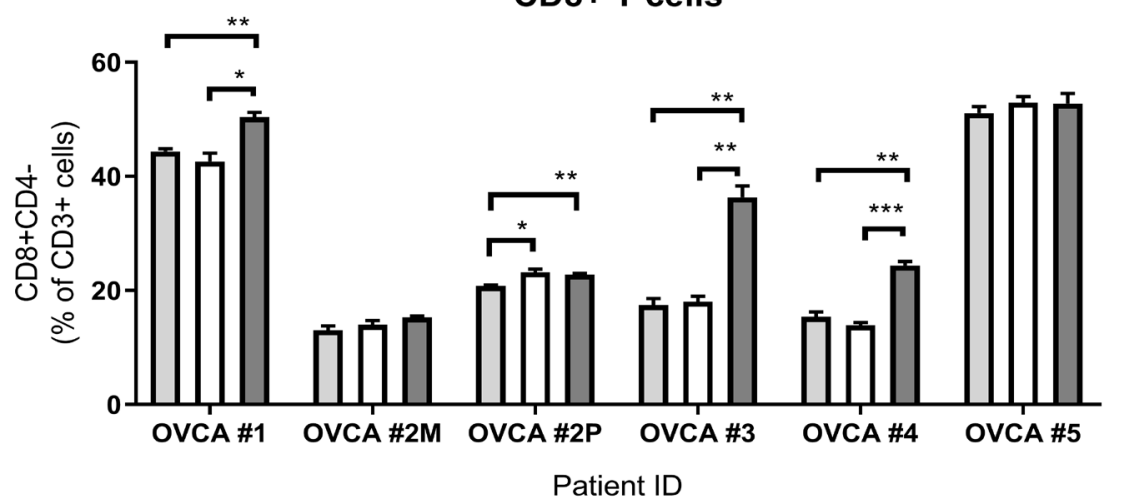

C

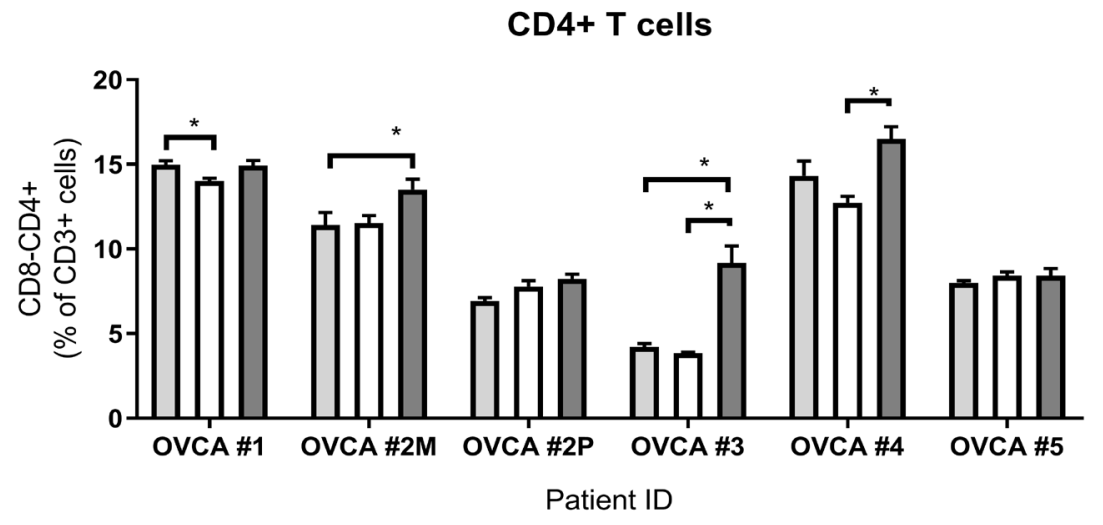

B

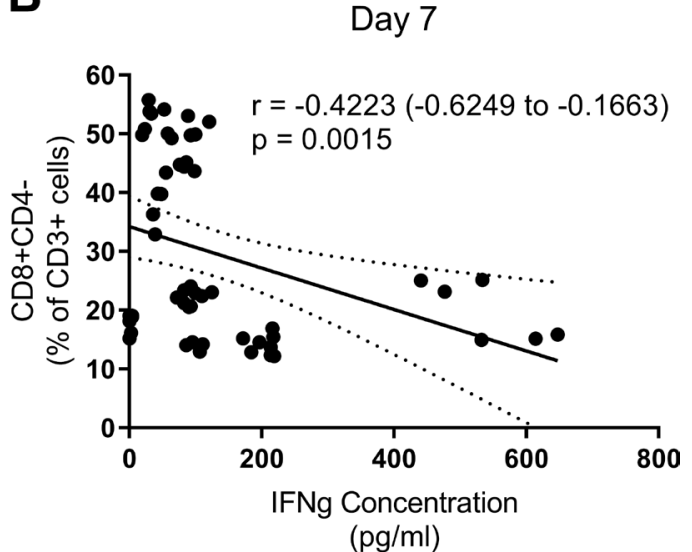

D

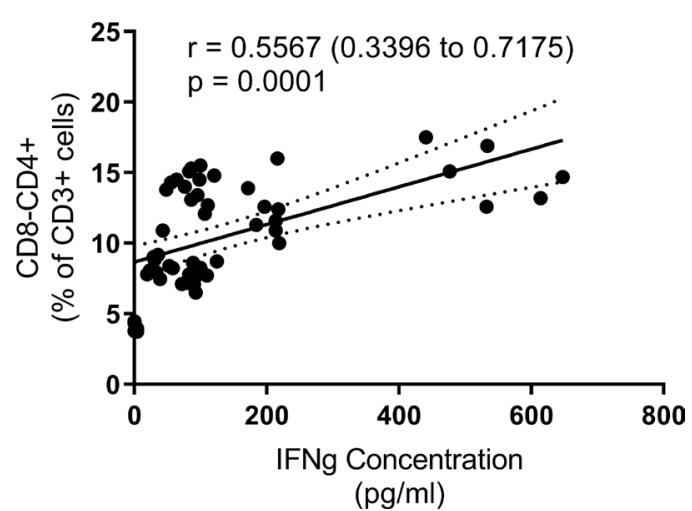

Figure 5 Presence of tumor-infiltrating lymphocytes (TIL) in autologous TIL-tumor cocultures and the relation with interferon gamma (IFNg) levels at day 7. Cells were collected from autologous TIL-tumor cocultures for characterization at day 7. (A) CD4-CD8+ T cells, (B) correlation between CD4-CD8+ T cells and IFNg levels, (C) CD4+CD8T cells, (D) correlation between CD4+CD8T cells and IFNg levels. Each patient experiment was performed in triplicate and data are shown as mean+SEM. Statistical significance is represented as ${ }^{\star} \mathrm{p}<0.05,{ }^{* \star} \mathrm{p}<0.01$ and ${ }^{\star \star *} \mathrm{p}<0.001$. OVCA, ovarian cancer.

was found on OVCA \#2P (figure 5C). Ad5/3-E2F-D24hTNFa-IRES-hIL2 therapy statistically reduced the presence of CD4+CD27+ TILs in OVCA \#2M compared with the Ad5/3-E2F-D24 group (online supplementary figure 3D). In contrast with the CD8+ TIL fraction, the concomitant increase in the presence of CD4+ TILsand IFNg levels appears to be strongly related $(\mathrm{r}=0.5567(0.3396$ to 0.7175$), \mathrm{p}=0.0001$; figure $5 \mathrm{D})$. A weaker correlation was also found between the increase in CD16+CD56+ NK cells and IFNg levels in the coculture $(r=0.3678(0.1030$ to $0.5840), p=0.0062)$. However, on treatment with Ad5/3E2F-D24-hTNFa-IRES-hIL2, only a trend (not statistically significant) for increased levels of NK cells was observed in all samples except OVCA \#4 (online supplementary figure $3 \mathrm{E}$ ).

\section{Frequency of PD-L1 in tumor cells and PD-1 in CD4+ TILs} influences the magnitude of antitumor TIL reactivity

Because we observed high variability in the magnitude of IFNg responses among the different patient samples, we hypothesized that the frequency of cells expressing PD-1/PD-L1 checkpoint molecules could explain this phenomenon. We first examined the frequency and expression of PD-L1 in tumor cells from ex vivo tumor cultures (online supplementary figure 4A). Except for OVCA\#4, there was an overall trend for reduced frequency of PD-L1+ tumor cells after virus treatment on day 3. On this day, PD-L1 expression was reduced in OVCA \#3 with both virus and in OVCA \#5 with Ad5/3-E2F-D24-hTNFaIRES-hIL2. A slight increase in PD-L1 expressing cells was seen mostly in Ad5/3-E2F-D24 treated cultures in OVCA \#2M and OVCA \#4. After 6 days of culturing, treatment of OVCA \#2M with Ad5/3-E2F-D24-hTNFa-IRES-hIL2 increased the frequency of PD-L1+ tumor cells relative to control groups. In contrast, most samples showed either no variation (OVCA \#2P) in the frequency of these cells or reduction of PD-L1+ tumor cells (OVCA \#3, \#4, \#5). Surprisingly, increased expression of PD-L1+ tumor cells was seen in OVCA \#2M, OVCA \#2P and OVCA \#5 (to some extent) treated with viruses, while a reduction in PD-L1 expression was observed in OVCA \#3 and OVCA \#4. 
A
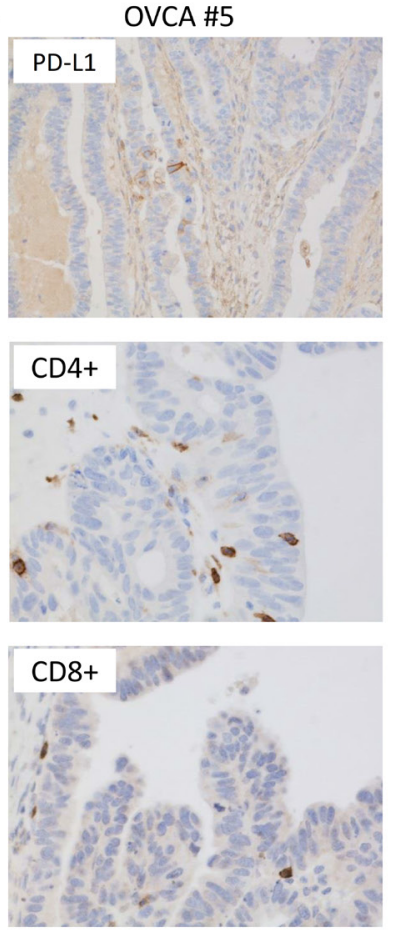

OVCA \#2
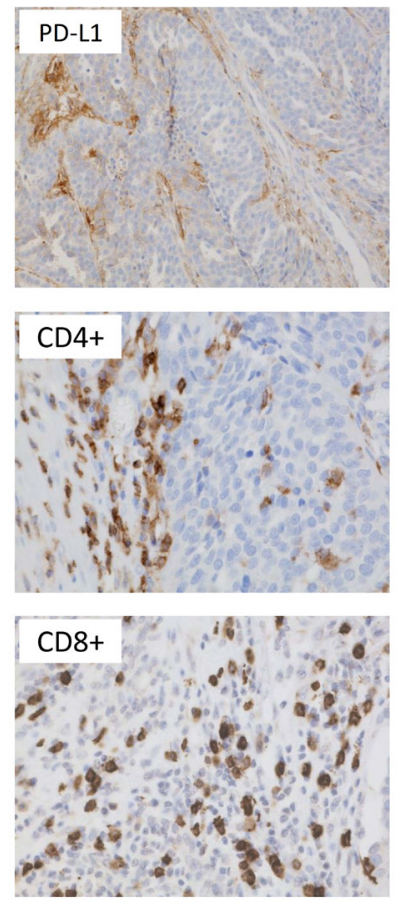

B

\section{.}

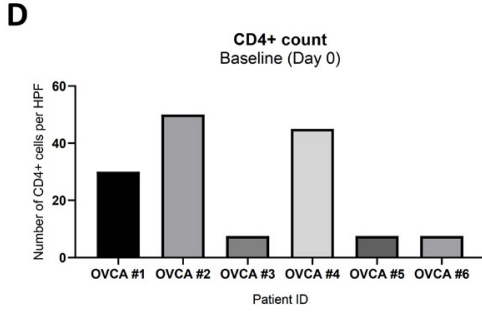

F

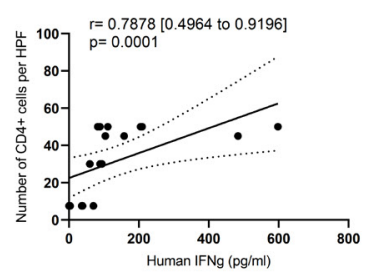

C

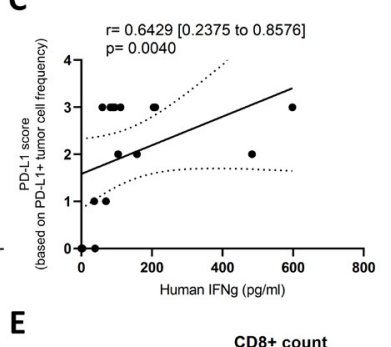

E

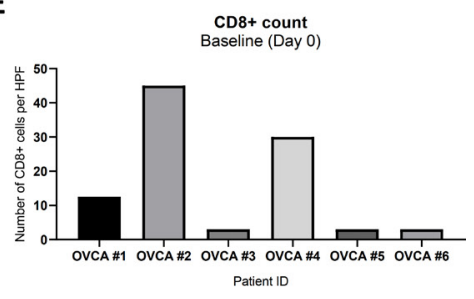

G

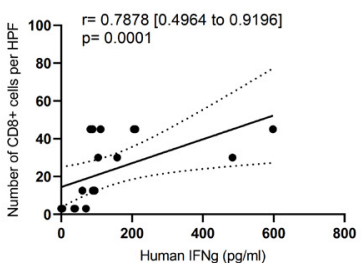

Figure 6 Assessment of programmed death ligand 1 (PD-L1)+, CD4+ and CD8+ cell frequency in immunohistochemistrystained tissues and its relation with day 7 interferon gamma (IFNg) response in cocultures of clinically relevant tumor-infiltrating lymphocytes (TIL) and autologous T-cell-depleted single-cell suspensions. After resection, ovarian cancer samples were fixed, paraffin embedded and stained for different markers by immunohistochemistry. (A) Representative image of a patient sample with low (OVCA \#5) or high (OVCA \#2) PD-L1 expression, CD4 and CD8 count (all stained in brown), (B) PD-L1 score in tumor cells, (C) correlation between PD-L1 in cancer cells and day 7 IFNg response in cocultures, (D) CD4+ count per high-power field (HPF), (E) CD8+ count per HPF, (F) correlation between CD4+ count per HPF and day 7 IFNg response in cocultures and (G) correlation between CD8+ count per HPF and day 7 IFNg response in cocultures. The PD-L1 score is as follows: $0=<1 \%$, $1=1 \%-4 \%, 2=5 \%-9 \%, 3=10 \%-49 \%, 4=>50 \%$ of PD-L1+ tumor cells. CD8+ and CD4+ cells were counted using high-power field at $400 \times$.

Evaluation of PD-L1 expression, and CD4 and CD8 count in baseline tumors (after resection) was done with immunohistochemistry (figure 6A). Low PD-L1 expressing tumors (such as OVCA \#5) were also poorly infiltrated by CD4+ andCD8+ cells as opposed to high PD-L1 expressing tumors where this was not observed (such as OVCA \#2) seen (figure 6A). Baseline OVCA \#1, \#2 and \#4 tumor samples scored the highest for baseline PD-L1 expression (figure 6B) and a strong positive correlation was found between these scores and day 7 IFNg response of cocultures of clinically relevant TILs with autologous T-cell single-cell suspensions at day $7 \quad(\mathrm{r}=0.6429$ [0.2375 to 0.8576 ] $\mathrm{p}=0.0040$; figure $6 \mathrm{C}$ ). Following the same trend, these patient samples also exhibited high numbers of $\mathrm{CD} 4+$ cells (figure 6D), and, to a lower extent, CD8+ cells (figure 6E). Both subset counts showed also a significant positive correlation with the day 7 IFNg response in cocultures (both $\mathrm{r}=0.7878$ (0.4964 to 0.9196 ), $\mathrm{p}=0.0001$; figure $6 \mathrm{~F}, \mathrm{G})$.

When we looked at CD4+PD-1+ T cells in day 14-expanded TILs, their frequency was lowest in OVCA \#2M and OVCA \#4 (marginally; online supplementary figure 5A), and this negatively influenced the IFNg response observed in different patient cocultures $(\mathrm{r}=-0.4859 \quad(-0.7825$ to
$-0.009699), \mathrm{p}=0.0409$; online supplementary figure 5B). CD8+PD-1+ TILs were observed at lower levels among different patient TILs (online supplementary figure 5C) and no relation was found between IFNg responses and their frequency in the latter ( $r=-0.3104(-0.6869$ to 0.1974$)$, $\mathrm{p}=0.21$; online supplementary figure $5 \mathrm{D})$.

\section{DISCUSSION}

Despite the existence of multiple immune targets in the tumor microenvironment of OVCA, consistent clinical efficacy is rarely achieved when immunotherapies are used. In particular, TIL-based ACT has demonstrated discrepant results in patients with OVCA, which may reflect differences in patient inclusion criteria or the clinical protocol.

For instance, the high response rates $(82 \%)$ achieved in a clinical study by Aoki and colleagues may be due to the inclusion of a patient cohort administered with TILs as first-line therapy, with high-dose cyclophosphamide, which is active in chemotherapy-naïve OVCA. The authors also had another cohort which received concurrent cisplatin-based chemotherapy. ${ }^{31}$ Similarly, in another clinical study, 100\% 3-year survival was achieved when the 
infusion of TILs was performed after debulking surgery and adjuvant chemotherapy. ${ }^{32}$ The survival of matched controls was $67.5 \%$.

In contrast, a recent clinical trial has demonstrated that TIL-treated patients with chemotherapy-refractory OVCA saw tumor progression after short-term stable disease, suggesting that the tumor microenvironment may be responsible for restricting antitumor responses mediated by TILs. ${ }^{10}$

In the present study, we demonstrate that Ad5/3-E2FD24-hTNFa-IRES-hIL2 improves the immunological activation of TILs obtained from patients with OVCA. Primarily, these observations were possible because our pseudotyped (Ad5/3) oncolytic adenovirus platform possesses distinct properties that are highly attractive for the treatment of OVCAs. For instance, the fact that we have retargeted serotype 5 adenoviruses to serotype 3 receptors allows the entry of Ad5/3 adenoviruses through receptors known to be highly upregulated in OVCA cells. $^{27}$ This has been shown to result in efficient transgene delivery and good antitumor efficacy in preclinical studies $^{27}$ and clinical reports ${ }^{21}$ involving this malignancy.

The use of Ad5/3-E2F-D24 or Ad5/3-E2F-D24hTNFa-IRES-hIL2 in our ex vivo tumor culture system demonstrated effective oncolytic capability despite the differences in the origin or subtype of the patient's OVCA cells. Interestingly, the virus replication variability previously seen in patients treated with Ad5/3 oncolytic viruses could be (in part) explained by these differences. Yet, to date, these hypotheses have not been explored in detail. It is important to note, however, that OVCA cells feature an aberrant $\mathrm{p} 16 / \mathrm{Rb}$ pathway leading to an accumulation of intracellular free E2F proteins. ${ }^{33}$ The fact that both viruses make use of an E2F promoter, should allow virus replication regardless of differences of histology. Moreover, the presence of transgenes in Ad5/3E2F-D24-hTNFa-IRES-hIL2 did not hinder oncolysis of ovarian tumor cells. This concurs with the tumor cell killing profile that we previously observed with this virus in OVCA cell lines and in vivo in an orthotopic model. ${ }^{20}$ In addition, this observation is particularly useful considering that unarmed oncolytic adenoviruses seem to result in inferior immunological benefit in patients with ovarian tumors. ${ }^{34}$ However, Ad5/3-E2F-D24-hTNFa-IRES-hIL2 showed an apparent lack of enhanced efficacy over the parental backbone in this ex vivo tumor culture system. Notably this is not observed in vivo, where treatment with Ad5/3-E2F-D24-hTNFa-IRES-hIL2 demonstrates a clear superior curative efficacy over Ad5/3-E2F-D24 in HapT1 (hamster pancreatic cancer) tumor-bearing hamsters. ${ }^{20}$ Indeed, evaluation of oncolysis of immune-stimulating oncolytic viruses in this culture system, where the tumor microenvironment is present, could represent a potential pitfall. Because the majority of patient samples contained a high frequency of tumor cells, we determined overall tumor viability based on mitochondrial activity of the well. Given the T-cell stimulatory properties of Ad5/3E2F-D24-hTNFa-IRES-hIL2, the possibility that the readout could have been influenced by T-cell proliferation cannot be discarded. Thus, one could hypothesize that experiments with a more sensitive assay could better highlight the efficacy differences between Ad5/3-E2FD24-hTNFa-IRES-hIL2 and Ad5/3-E2F-D24. Importantly, it appears Ad5/3-E2F-D24-hTNFa-IRES-hIL2 can achieve superior immunological activation without loss of oncolytic potency.

In order to sustain durable tumor regressions, TILs must be capable of mounting a potent antitumor response and sustaining it with proinflammatory factors. This situation does not seem to occur readily in the ovarian tumor microenvironment, as it is frequent that TILs are unable to produce cytokines with cytotoxic effects, such as TNFa. ${ }^{35}$ Intriguingly, intraperitoneal administration of IL-2 is able to induce partial responses in patients with OVCA. This suggests that high levels of IL-2 are required at the tumor site to disrupt tolerance towards ovarian tumors. ${ }^{36}$

Concurring with this notion, Ad5/3-E2F-D24-hTNFaIRES-hIL2 treatment of ovarian ex vivo tumor cultures increased the production of IFNg, a cytokine characteristic of heightened TIL activity. Concomitant increase of CXCL10, a chemokine involved in TIL trafficking and OVCA arrest in mice, ${ }^{37}$ is concordant with SPECT/CT data from mice and hamsters generated by our group. These studies showed increased infiltration of transferred $\mathrm{T}$ cells in murine melanoma and hamster pancreatic tumors injected with adenoviruses coding for TNFa and IL-2. ${ }^{22} 38$

Interestingly, oncolytic adenovirus therapy did not stimulate the production of high levels of IFNb in ex vivo tumor cultures, which suggests that antiviral defenses are not easily induced in the immunosuppressive OVCA microenvironment. In line with these observations, de Queiroz and colleagues have recently reported that OVCA cell lines often present defects in viral sensing mechanisms, which contribute to higher susceptibility to oncolytic virus therapy. ${ }^{39}$ Slower induction of antiviral immunity could offer some advantages to oncolytic virus therapy, if the virus is able to replicate more. Yet, since we have previously observed that type I IFN pathways can induce resistance to Ad5/3 oncolytic adenovirus therapy in an in vivo human OVCA xenograft model, ${ }^{40}$ it is also possible that increased immune stimulation could increase antiviral responses.

Of note, the reduction of suppressive cytokines (IL-6, TGF-b1 and arginase) on Ad5/3-E2F-D24-hTNFaIRES-hIL2 therapy is particularly relevant, considering that increased levels of these cytokines are implicated in the suppression of antitumor TIL activity in a number of malignancies, including OVCA. ${ }^{41}$ In particular, TGF-b1 is reported to increase the invasive potential of OVCA cells. ${ }^{42}$ Besides the negative effects on the adaptive immune system, such cytokines are a functional signature of infiltrating MDSCs and Tregs. ${ }^{41}$ Decreased activity of MDSCs is in line with previous observations where intratumororal injections of TNFa and IL-2-coding 
adenovirus, combined with T-cell therapy, led to a reduction in functional MDSCs in mice developing spontaneous melanoma. ${ }^{43}$

Skewing of the cytokine milieu towards Th1 signals is indicative of an ongoing inflammatory antitumor response, which in OVCA is predominantly associated with CD8+ and CD4+ TIL activity. ${ }^{41}$ In a recent study, Owens and colleagues found that CD4+CD69+ TILs are capable of inducing high levels of Th1 cytokines which may explain the tight positive correlation between CD69 expression in CD4+ TILs, and the levels of immune stimulatory cytokines found in cultures treated with Ad5/3-E2F-D24-hTNFa-IRES-hIL2. ${ }^{44}$ This observation is appealing since active CD4+ TILs predict improved clinical outcome, ${ }^{45}$ supporting the use of Ad5/3-E2F-D24hTNFa-IRES-hIL2 in this indication.

On another note, the decrease in the frequency of $\mathrm{CD} 4+\mathrm{CD} 25+\mathrm{T}$ cells in cultures treated with virus could indicate a drop in recently activated TILs. However, the effects of virus-derived IL-2 are noticeable as CD25 expression remained high in most patient cultures treated with Ad5/3-E2F-D24-hTNFa-IRES-hIL2. Considering that CD25 expression decreases after its peak at 72 hours, ${ }^{46}$ it is conceivable that IL-2-coding oncolytic adenoviruses are capable of ensuring continuous activation of T cells.

The generation and expansion of TILs for therapeutic application can be achieved with high reproducibility in different tumor types, ${ }^{4}$ and OVCA is no exception. A recent study reported that TIL culture resulted in grafts with a high CD4-to-CD8 cell ratio. ${ }^{47}$ In contrast, we and others have frequently seen the inverse situation, perhaps reflecting variations in the TIL production protocols, ${ }^{44}$ such as the concentration of IL-2 used. $^{47}$

In ACT with TILs, IL-2 is used for the in vivo maintenance of the TIL graft activity. ${ }^{4}$ Thus, production of IL-2 by Ad5/3-E2F-D24-hTNFa-IRES-hIL2 may explain the increase of TIL-derived IFNg seen (figure 4). In the context of immunotherapy of OVCA, IFNg production is beneficial since it has been shown by others to promote the upregulation of antigen presentation machinery and induce direct apoptosis in OVCA tumor cells. ${ }^{48}$

Interestingly, as noted in melanoma and OVCA, ${ }^{44} 47$ our data suggest that the CD4+ fraction may contain potent tumor-reactive T cells. In line with this concept, Pedersen and colleagues also reported high expression of human leucocyte antigen class II in OVCA patient biopsies, suggesting the involvement of $\mathrm{CD} 4+\mathrm{T}$ cells in antitumor immunity in this indication. ${ }^{10}$ Cytotoxic CD4+ T cells also play a major role in eliminating virus-infected cells ${ }^{49}$ and, since they can be present in the expanded TIL product, they could have had played a role in the reactivity seen here.

Notably, there was high variability in the magnitude of TIL-IFNg responses across different patients although this was not influenced by the unwanted frequency of Treg TIL cultures. In agreement, indirect evidence shows that treatment of patients with metastatic melanoma using adoptive transfer with enriched 'young' CD8+ TILs did not result in significant clinical benefit in comparison with unselected 'young' TILs. ${ }^{50}$ It appears, however, that reconstitution of endogenous Tregs during IL-2 postconditioning is a key driver for the negative outcome of ACT with TILs. ${ }^{51}$

Recently, the PD-1/PD-L1 axis has drawn extensive attention due to its role in the negative regulation of antitumor immune responses in many tumor types, including OVCA. $^{52}$ Indeed, expression of PD-L1 at the tumor and PD-1 in TILs has been deemed to compromise the efficacy of adoptive TIL therapy of patients with OVCA in a recent clinical trial. ${ }^{10}$ In high-grade serous OVCA, PD-L1+ cells (containing also tumor cells) are positively associated with infiltration of TILs, thus providing a favorable prognostic factor. ${ }^{53}$ This is not surprising since PD-L1 expression in tumors also defines a previously immunologically active tumor, ${ }^{54}$ which has developed the ability to silence adaptive immunity. Additionally, the fact that the frequency of CD4+PD-1+ TILs, not CD8+PD-1+ TILs, negatively influences the IFNg response, reinforces the importance of CD4+ TILs in the antitumor response in OVCA. Further experiments would be required to detail the exact mechanisms of this immunological response. Together with our data, these findings may explain the susceptibility of some samples to immunotherapy with expanded TILs. It is important to note that even though the frequency of PD-L1+ tumor cells and CD4+PD-1+ TILs may determine the outcome of T-cell-based immunotherapy, Ad5/3-E2FD24-hTNFa-IRES-hIL2 was able unleash significant TIL antitumor responses independently of these factors.

Perhaps the biggest challenge with using patientderived ex vivo tumor cultures to study the immunological potency of oncolytic adenoviruses is the absence of a full immune system. The fact that this is an isolated culture system under-represents the dynamics of adaptive immune responses. Influx of $\mathrm{T}$ cells is not possible and generation of new T-cell responses is confined to the local repertoire of antigen-presenting cells potentially skewing the efficacy of cytokine-coding adenovirus aiming at stimulating T cells. Additionally, this system lacks a 3D structure preventing us from determining the real kinetics of virus dissemination. It is important, however, to put these flaws in the context of the available tools to study immune-stimulating oncolytic adenoviruses in OVCA. First, oncolytic adenoviruses only fully replicate in human and, partially, in Syrian hamsters (Mesocricetus auratus). While it is relatively easy to establish human tumor xenografts (not patient derived) in immunocompromised mice, it lacks an immune system, thus only virus replication can be studied in this model. On the other hand, we could not use Syrian hamsters because, to date, the species lacks an OVCA tumor model and reagents that could allow us to gain further insight into the immunological impact of our oncolytic adenovirus platform. On the other hand, immunocompetent mouse models offer an incomplete vision on the immunological effects of oncolytic adenovirus because, while cytokine transgene expression is possible, lytic replication is not achieved. 
Hence, ex vivo tumor cultures offer a valuable method to determine in-depth characterization of the local effects of immune-stimulating adenoviruses in human substrates while taking into account the heterogeneity of the human tumors.

\section{CONCLUSIONS}

In conclusion, while Ad5/3 chimeric oncolytic adenoviruses in general provide an efficacious treatment modality for OVCA, ${ }^{1721}$ our findings indicate that the introduction of TNFa and IL-2 is appealing for promotion of substantial immunological activation of locoregional TILs. Increase in CXCL10 suggests that local virus replication can also recruit $\mathrm{T}$ cells from further away compartments, such as the blood, for example. Furthermore, the use of oncolytic adenoviruses coding for TNFa and IL-2 reconfigures the ovarian tumor microenvironment to enhance the tumor reactivity of clinically relevant OVCA TILs.

Together with the enhanced in vivo efficacy observed by us and others, ${ }^{20}{ }^{24}$ these data strengthen the rationale for a clinical trial where Ad5/3-E2F-D24-hTNFa-IRES-hIL2 would be administered in patients with OVCA receiving TIL therapy, in order to improve clinical responses. Incurable OVCA frequently remains intraperitoneal, which creates a strong rationale for intraperitoneal delivery. The peritoneal cavity can be seen as a large immunological organ..$^{55}$ These features make OVCA an appealing target for oncolytic immunotherapy.

\section{Author affiliations \\ ${ }^{1}$ Cancer Gene Therapy Group, Translational Immunology Research Program, Faculty of Medicine, University of Helsinki, Helsinki, Finland \\ ${ }^{2}$ TILT Biotherapeutics, Helsinki, Finland \\ ${ }^{3}$ Helsinki University Hospital Comprehensive Cancer Center, Helsinki, Finland ${ }^{4}$ Cancer Center Amsterdam, Departments of Medical Oncology and Radiation Oncology, University Medical Center Amsterdam, Amsterdam, The Netherlands ${ }^{5}$ Department of Obstetrics and Gynecology, Helsinki University Hospital, Helsinki, Finland}

Acknowledgements We thank Minna Oksanen, Susanna Grönberg-Vähä-Koskela, Riikka Kalliokoski, Helena Taskinen, Reija Mia Kero, Randen-Brady, the tissue preparation and histochemistry unit of the University of Helsinki and the department of pathology of the HUS lab for the expert assistance.

Contributors JMS, $\mathrm{CH}, \mathrm{VCC}, \mathrm{RH}, \mathrm{MS}$, TdG and $\mathrm{AH}$ designed the experiments. JMS, CH, VCC, DCAQ and SZ conducted the experiments. JMS and RB analyzed the results. AK and $\mathrm{HL}$ collected the patient samples. All the authors contributed to writing and reviewing the manuscript.

Funding This study was supported by the European Commission Marie Curie Innovative Training Network (ITN) grant VIRION (H2020-MSCA-ITN-2014, project number 643130), Finnish Cultural Foundation, Jane and Aatos Erkko Foundation, HUCH Research Funds (EV0), Sigrid Juselius Foundation, Finnish Cancer Organizations, University of Helsinki, The Finnish Society of Sciences and Letters and TILT Biotherapeutics.

Competing interests $\mathrm{AH}$ is a shareholder in Targovax ASA. AH is an employee and shareholder in TILT Biotherapeutics. JMS, VCC, MS and RH are employees of TILT Biotherapeutics.

Patient consent for publication Not required.

Ethics approval Central Hospital Operative Ethics Committee approved the studies (permit number 120/13/03/02/16) performed on patient material and written informed consent was given by the patients.

Provenance and peer review Not commissioned; externally peer reviewed.
Data availability statement Data are available upon reasonable request.

Open access This is an open access article distributed in accordance with the Creative Commons Attribution Non Commercial (CC BY-NC 4.0) license, which permits others to distribute, remix, adapt, build upon this work non-commercially, and license their derivative works on different terms, provided the original work is properly cited, appropriate credit is given, any changes made indicated, and the use is non-commercial. See http://creativecommons.org/licenses/by-nc/4.0/.

ORCID iD

Akseli Hemminki http://orcid.org/0000-0001-7103-8530

\section{REFERENCES}

1 Medler TR, Cotechini T, Coussens LM. Immune response to cancer therapy: mounting an effective antitumor response and mechanisms of resistance. Trends Cancer 2015;1:66-75.

2 Sato E, Olson SH, Ahn J, et al. Intraepithelial CD8+ tumor-infiltrating lymphocytes and a high CD8+/regulatory $T$ cell ratio are associated with favorable prognosis in ovarian cancer. Proc Natl Acad Sci U S A 2005;102:18538-43.

3 Gooden MJM, de Bock GH, Leffers N, et al. The prognostic influence of tumour-infiltrating lymphocytes in cancer: a systematic review with meta-analysis. Br J Cancer 2011;105:93-103.

4 Rosenberg SA, Restifo NP. Adoptive cell transfer as personalized immunotherapy for human cancer. Science 2015;348:62-8.

5 Andersen R, Donia M, Ellebaek E, et al. Long-Lasting complete responses in patients with metastatic melanoma after adoptive cell therapy with tumor-infiltrating lymphocytes and an attenuated IL2 regimen. Clinical Cancer Research 2016;22:3734-45.

6 Dudley ME, Yang JC, Sherry R, et al. Adoptive cell therapy for patients with metastatic melanoma: evaluation of intensive myeloablative chemoradiation preparative regimens. Journal of Clinical Oncology 2008;26:5233-9.

7 Rosenberg SA, Yang JC, Sherry RM, et al. Durable complete responses in heavily pretreated patients with metastatic melanoma using T-cell transfer immunotherapy. Clinical Cancer Research 2011;17:4550-7.

8 Aoki Y, Takakuwa K, Kodama S, et al. Use of adoptive transfer of tumor-infiltrating lymphocytes alone or in combination with cisplatincontaining chemotherapy in patients with epithelial ovarian cancer. Cancer Res 1991:51:1934-9.

9 Freedman RS, Edwards CL, Kavanagh JJ, et al. Intraperitoneal adoptive immunotherapy of ovarian carcinoma with tumor-infiltrating lymphocytes and low-dose recombinant interleukin-2: a pilot trial. $J$ Immunother Emphasis Tumor Immunol 1994;16:198-210.

10 Pedersen M, Westergaard MCW, Milne K, et al. Adoptive cell therapy with tumor-infiltrating lymphocytes in patients with metastatic ovarian cancer: a pilot study. Oncoimmunology 2018;7:e1502905.

11 Andersen R, Donia M, Westergaard MCW, et al. Tumor infiltrating lymphocyte therapy for ovarian cancer and renal cell carcinoma. Hum Vaccin Immunother 2015;11:2790-5.

12 Santoiemma PP, Powell DJ. Tumor infiltrating lymphocytes in ovarian cancer. Cancer Biol Ther 2015;16:807-20.

13 Cui TX, Kryczek I, Zhao L, et al. Myeloid-Derived suppressor cells enhance stemness of cancer cells by inducing microRNA101 and suppressing the corepressor CtBP2. Immunity 2013;39:611-21.

14 Curiel TJ, Coukos G, Zou L, et al. Specific recruitment of regulatory $T$ cells in ovarian carcinoma fosters immune privilege and predicts reduced survival. Nat Med 2004;10:942-9.

15 Seder RA, Darrah PA, Roederer M. T-Cell quality in memory and protection: implications for vaccine design. Nat Rev Immunol 2008;8:247-58.

16 Cervera-Carrascon V, Havunen R, Hemminki A. Oncolytic adenoviruses: a game changer approach in the battle between cancer and the immune system. Expert Opin Biol Ther 2019;19:443-55.

17 Kanerva A, Nokisalmi P, Diaconu I, et al. Antiviral and antitumor T-cell immunity in patients treated with GM-CSF-coding oncolytic adenovirus. Clin Cancer Res 2013;19:2734-44.

18 Woller N, Gürlevik E, Fleischmann-Mundt B, et al. Viral infection of tumors overcomes resistance to PD-1-immunotherapy by broadening Neoantigenome-directed T-cell responses. Molecular Therapy 2015;23:1630-40.

19 Tähtinen S, Grönberg-Vähä-Koskela S, Lumen D, et al. Adenovirus improves the efficacy of adoptive T-cell therapy by recruiting immune cells to and promoting their activity at the tumor. Cancer Immunol Res 2015;3:915-25. 
20 Havunen R, Siurala M, Sorsa S, et al. Oncolytic adenoviruses armed with tumor necrosis factor alpha and interleukin-2 enable successful adoptive cell therapy. Mol Ther Oncolytics 2017;4:77-86.

21 Kanerva A, Koski A, Liikanen I, et al. Case-Control estimation of the impact of oncolytic adenovirus on the survival of patients with refractory solid tumors. Mol Ther 2015;23:321-9.

22 Siurala M, Havunen R, Saha D, et al. Adenoviral delivery of tumor necrosis factor- $\alpha$ and interleukin-2 enables successful adoptive cell therapy of immunosuppressive melanoma. Mol Ther 2016;24:1435-43.

23 Tähtinen S, Kaikkonen S, Merisalo-Soikkeli M, et al. Favorable alteration of tumor microenvironment by immunomodulatory cytokines for efficient T-cell therapy in solid tumors. PLoS One 2015;10:e0131242.

24 Watanabe K, Luo Y, Da T, et al. Pancreatic cancer therapy with combined mesothelin-redirected chimeric antigen receptor $\mathrm{T}$ cells and cytokine-armed oncolytic adenoviruses. JCl Insight 2018;3.

25 Cervera-Carrascon V, Siurala M, Santos JM, et al. TNFa and IL-2 armed adenoviruses enable complete responses by anti-PD-1 checkpoint blockade. Oncoimmunology 2018;7:e1412902.

26 Cervera-Carrascon V, Quixabeira D, Munaro E, et al. Enabling checkpoint inhibitors with oncolytic viruses to deliver complete responses: a matter of timing. AACR Tumor Immunology and Immunotherapy meeting 2018.

27 Kanerva A, Zinn KR, Chaudhuri TR, et al. Enhanced therapeutic efficacy for ovarian cancer with a serotype 3 receptor-targeted oncolytic adenovirus. Mol Ther 2003;8:449-58.

28 Taipale K, Tähtinen ST, Havunen R, et al. Interleukin 8 activity influences the efficacy of adenoviral oncolytic immunotherapy in cancer patients. Oncotarget 2018;9:6320-35.

29 Koski A, Bramante S, Kipar A, et al. Biodistribution analysis of oncolytic adenoviruses in patient autopsy samples reveals vascular transduction of Noninjected tumors and tissues. Mol Ther 2015;23:1641-52.

30 Donia M, Junker N, Ellebaek E, et al. Characterization and Comparison of 'Standard' and 'Young' Tumour-Infiltrating Lymphocytes for Adoptive Cell Therapy at a Danish Translational Research Institution. Scand J Immunol 2012;75:157-67.

31 Aoki Y, Takakuwa K, Kodama S, et al. Use of adoptive transfer of tumor-infiltrating lymphocytes alone or in combination with cisplatincontaining chemotherapy in patients with epithelial ovarian cancer. Cancer Res 1991:51:1934.

32 Fujita K, Ikarashi H, Takakuwa K, et al. Prolonged disease-free period in patients with advanced epithelial ovarian cancer after adoptive transfer of tumor-infiltrating lymphocytes. Clin Cancer Res 1995;1:501-7.

33 Todd MC, Sclafani RA, Langan TA. Ovarian cancer cells that coexpress endogenous $\mathrm{Rb}$ and $\mathrm{p} 16$ are insensitive to overexpression of functional p16 protein. Oncogene 2000;19:258-64.

34 Pesonen S, Diaconu I, Cerullo V, et al. Integrin targeted oncolytic adenoviruses Ad5-D24-RGD and Ad5-RGD-D24-GMCSF for treatment of patients with advanced chemotherapy refractory solid tumors. Int J Cancer 2012;130:1937-47.

35 Matsuzaki J, Gnjatic S, Mhawech-Fauceglia P, et al. Tumor-infiltrating NY-ESO-1-specific CD8 ${ }^{+} T$ cells are negatively regulated by LAG3 and PD-1 in human ovarian cancer. Proc Natl Acad Sci U S A 2010:107:7875-80.

36 Vlad AM, Budiu RA, Lenzner DE, et al. A phase II trial of intraperitoneal interleukin-2 in patients with platinum-resistant or platinum-refractory ovarian cancer. Cancer Immunology, Immunotherapy 2010;59:293-301.
$37 \mathrm{~K}$ Au K, Peterson N, Truesdell P, et al. Cxcl10 alters the tumour immune microenvironment and disease progression in a syngeneic murine model of high-grade serous ovarian cancer. Gynecol Oncol 2017:145:436-45.

38 Havunen R, Santos JM, Sorsa S, et al. Abscopal effect in Noninjected tumors achieved with Cytokine-Armed oncolytic adenovirus. Molecular Therapy - Oncolytics 2018:11:109-21.

39 de Queiroz NMGP, Xia T, Konno H, et al. Ovarian cancer cells commonly exhibit defective sting signaling which affects sensitivity to viral oncolysis. Mol Cancer Res 2019;17:974-86.

40 Liikanen I, Monsurrò V, Ahtiainen L, et al. Induction of interferon pathways mediates in vivo resistance to oncolytic adenovirus. Molecular Therapy 2011;19:1858-66.

41 McCloskey C, Rodriguez G, Galpin K, et al. Ovarian cancer immunotherapy: preclinical models and emerging therapeutics. Cancers 2018;10:244.

42 Yeung T-L, Leung CS, Wong K-K, et al. TGF- $\beta$ modulates ovarian cancer invasion by upregulating CAF-derived versican in the tumor microenvironment. Cancer Res 2013;73:5016-28.

43 Tähtinen S, Blattner C, Vähä-Koskela M, et al. T-Cell therapy enabling adenoviruses coding for IL2 and TNF $\alpha$ induce systemic immunomodulation in mice with spontaneous melanoma. Journal of Immunotherapy 2016;39:343-54.

44 Owens GL, Price MJ, Cheadle EJ, et al. Ex vivo expanded tumourinfiltrating lymphocytes from ovarian cancer patients release antitumour cytokines in response to autologous primary ovarian cancer cells. Cancer Immunology, Immunotherapy 2018;67:1519-31.

45 Tsiatas ML, Gyftaki R, Liacos C, et al. Study of T lymphocytes infiltrating peritoneal metastases in advanced ovarian cancer: associations with vascular endothelial growth factor levels and prognosis in patients receiving platinum-based chemotherapy. Int J Gynecol Cancer 2009;19:1329-34.

46 Leonard WJ, Krönke M, Peffer NJ, et al. Interleukin 2 receptor gene expression in normal human T lymphocytes. Proc Natl Acad Sci U S A 1985;82:6281-5.

47 Westergaard MCW, Andersen R, Chong C, et al. Tumour-Reactive T cell subsets in the microenvironment of ovarian cancer. $\mathrm{Br} J$ Cancer 2019;120:424-34.

48 Wall L, Burke F, Barton C, et al. Ifn-Gamma induces apoptosis in ovarian cancer cells in vivo and in vitro. Clin Cancer Res 2003;9:2487-96.

49 Muraro E, Merlo A, Martorelli D, et al. Fighting viral infections and virus-driven tumors with cytotoxic CD4+ T cells. Front Immunol 2017;8.

50 Dudley ME, Gross CA, Somerville RPT, et al. Randomized selection design trial evaluating CD8+-enriched versus unselected tumorinfiltrating lymphocytes for adoptive cell therapy for patients with melanoma. J Clin Oncol 2013;31:2152-9.

51 Yao X, Ahmadzadeh M, Lu Y-C, et al. Levels of peripheral CD4+Foxp3+ regulatory $T$ cells are negatively associated with clinical response to adoptive immunotherapy of human cancer. Blood 2012;119:5688-96.

52 Gaillard SL, Coleman RL. Identifying markers of immune response in ovarian cancer: does PD-L1 expression meet the mark? Annals of Oncology 2019;30:1025-8.

53 Webb JR, Milne K, Kroeger DR, et al. Pd-L1 expression is associated with tumor-infiltrating $\mathrm{T}$ cells and favorable prognosis in high-grade serous ovarian cancer. Gynecol Oncol 2016;141:293-302.

54 Chen DS, Mellman I. Elements of cancer immunity and the cancerimmune set point. Nature 2017;541:321-30.

55 Meza-Perez S, Randall TD. Immunological functions of the omentum. Trends Immunol 2017;38:526-36. 